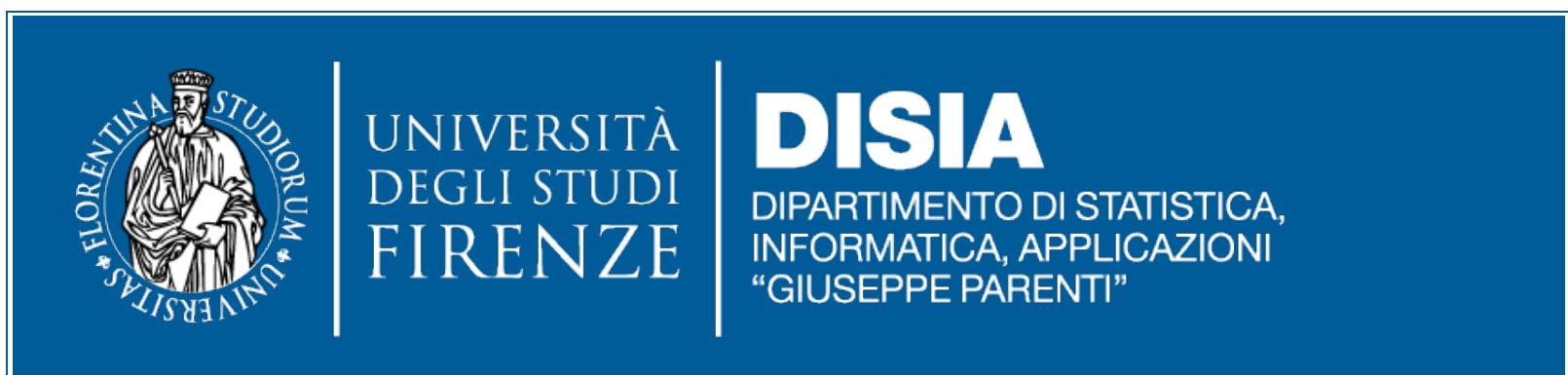

\title{
Realized variance modeling: decoupling forecasting from estimation
}

Fabrizio Cipollini, Giampiero M. Gallo, Alessandro Palandri

\section{DISIA WORKING PAPER $2019 / 05$}

(c) Copyright is held by the author(s). 


\title{
Realized variance modeling: decoupling forecasting from estimation
}

\author{
Fabrizio Cipollini ${ }^{1}$, Giampiero M. Gallo ${ }^{2, *}$ and Alessandro Palandri ${ }^{1,3}$ \\ ${ }^{1}$ Dipartimento di Statistica, Informatica, Applicazioni (DiSIA) \\ G. Parenti, Università di Firenze \\ ${ }^{2}$ Italian Court of Audits, and New York University in Florence \\ ${ }^{3}$ DCU Business School, Dublin City University
}

This Version: June 25, 2019

\begin{abstract}
In this paper we evaluate the in-sample fit and out-of-sample forecasts of various combinations of realized variance models and estimation criteria. Our empirical findings highlight that: independently of the econometrician's forecasting loss function, certain estimation criteria perform significantly better than others; the simple ARMA modeling of the log realized variance generates superior forecasts than the HAR family, for any of the forecasting loss functions considered; the $(2,1)$ parameterizations with negative lag- 2 coefficient emerge as the benchmark specifications generating the best forecasts and approximating long-run dependence as well as the HAR family.
\end{abstract}

Keywords: Variance modeling; Variance forecasting; Heterogeneous Autoregressive (HAR) model; Multiplicative Error Model (MEM); Realized variance

JEL classification: C32, C53, C58, G17

*Address correspondence to: Giampiero M. Gallo, NYU in Florence, Italy, Email: giampiero.gallo@nyu.edu. The views expressed in the article are those of the authors and do not involve the responsibility of the Corte dei conti. 


\section{Introduction}

The technical capability of recording and storing intradaily data has given a tremendous boost to the literature both on measurement issues (various forms of aggregation of ultra-high frequency data into a daily value) and on modeling their dynamics (extending the autoregressive flavor behind the GARCH class of models). There exist recent refinements within a somewhat consolidated menu of realized variance choices (plain vanilla, robustness to market microstructure noise, jumps, etc.; cf. Park and Linton, 2012, for a survey); in this work we focus on the realized kernel variance (Barndorff-Nielsen et al., 2008) to investigate a series of aspects related to modeling and forecasting. We will take it either as such, or as its square root, or yet as its logarithm, but for the sake of simplicity we generically talk here about realized variance modeling.

In this paper we consider that evaluating forecasts out-of-sample (OOS) is a matter of subjective taste about how to judge the distance between a predicted outcome and the actual value. In this respect, several examples of forecasting loss functions are considered, with the idea that a particular a priori choice is a consequence of individual preferences, hence it is not subject to qualitative assessment. In order to produce forecasts, two other elements are important: the model specification (the equation reproducing conditional variance dynamics), and the in-sample (IS) estimation criterion, i.e. the distance between observed and fitted values delivering parameter estimates. As for the adoption of a model specification and of an estimation criterion, we take the view that there are no natural a priori choices, but they must be geared toward obtaining the best results in terms of the given forecasting loss. In this respect, we argue that the choice of the latter should not force the same function to be repeated as the estimation criterion.

A first contribution of the paper is to explore whether, for a given forecasting loss, the IS estimation criterion with the same functional form produces the best OOS results by specification or, rather, other estimation criteria are to be preferred. A second contribution is to investigate the capability of a popular model selection tool such as the BIC to identify the best OOS specification. Third, we compare the benchmark HAR specifications of Corsi (2009) and Andersen et al. (2007) to several realized variance models inspired by the functionals of successful GARCH parameterizations which, for the most part, correspond to the simple ARMA modeling of either realized variance, its square root or its logarithm. Across classes, here 
we focus on the core specifications that could be extended to accommodate several refinements (asymmetry, see Engle and Gallo (2006), jumps, see Andersen et al. (2007), measurement errors, see Bollerslev et al. (2016), etc.).

We explore these issues on a panel of 28 constituents of the Dow Jones 30 Index using daily realized kernel variance observations from January 2005 to December 2015. The sample is split into six 5-year IS periods; we estimate all combinations of specifications and estimation criteria on the first IS period, generating OOS static forecasts for the ensuing 1-year period, then we move the IS window by one year and repeat the procedure. Our results may be summarized as follows: there are forecasting loss functions not particularly apt to be repeated as estimation criteria; we find encouraging results for the BIC ability to identify ex-ante the best OOS specification; the ARMA modeling of the log realized variance provides the best IS and OOS results. In general, we identify the $(2,1)$ structure with a negative lag-2 coefficient to be a good parameterization. As a reading key to these results, we find it informative to relate the goodness of the OOS forecasts to the structure's capability to mimic long memory features: as a matter of fact, these specifications deliver a long memory approximation which is equivalent to that of the HAR family, but overall superior OOS forecasts across assets.

The paper is structured as follows. Section 2 discusses the forecasting losses employed to compare model forecasts and the estimation criteria used to estimate parameters. Section 3 presents the specifications for the variance dynamics and Section 4 the information criteria used for model selection. Data are introduced in Section 5 and results are presented in Section 6. In Section 7 we provide a general discussion on the estimation criteria and the $(2,1)$ parameterizations and their ability to approximate long memory. Section 8 concludes.

\section{Forecasting Loss and Estimation Criterion}

A loss function maps events onto real numbers according to an individual's preference orderings. When estimating, values of the unknown coefficients of a parametric model are obtained from the minimization of a loss function IS. When forecasting, the quality of a specification is assessed through the calculation of a loss function based on the distance between the predicted outcomes and the actual values, OOS. In what follows, we argue that the priority given to forecasting requires to define separately the forecasting loss and the estimation criterion. 
With the expression forecasting loss (henceforth FL) we refer to the loss function used to evaluate the model performance OOS. Due to its subjective nature, the forecasting loss does not satisfy any criteria other than representing an individual's preferences. With the term estimation criterion (henceforth EC) we refer to the objective function used to obtain the parameter estimates. In general, the choice of this function responds to some features: theoretical properties of the resulting estimator, tractability, etc. Distinguishing between forecast evaluation and model estimation allows us to investigate, empirically and in the context of variance modeling, whether the EC that coincides with the FL produces the best OOS results. For FL and EC we consider some of the most common functionals adopted in the literature to measure the distance between observed and predicted values. Prevailing measures are quadratic, which corresponds to least-squares estimation, and Kullback-Leibler, which corresponds to quasi-maximum likelihood estimation when the chosen density function is linear-exponential.

\subsection{Quadratic Distance}

We characterize the general quadratic forecasting loss by:

$$
\sum_{t=T_{1}+1}^{T_{2}}\left[f\left(R V_{t}\right)-f\left(\sigma_{t}^{2}\right)\right]^{2}
$$

where $t=T_{1}+1, \ldots, T_{2}$ is the OOS period and $f\left(\sigma_{t}^{2}\right) \equiv \mathbb{E}_{t-1}\left[f\left(R V_{t}\right)\right]$. Common choices for the monotonic function $f(\cdot)$ are the identity, the square-root and the logarithm, which lead to what we define as the LS, SDLS and LNLS forecasting losses, respectively. As an example, for the square-root function we have that the forecasting loss synthesizes the distance between $R V_{t}^{1 / 2}$ and $\sigma_{t} \equiv E_{t-1}\left[R V_{t}^{1 / 2}\right], t=T_{1}+1, \ldots, T_{2}$.

We define the quadratic estimation criterion in the same way but without constraining it to have the same functional $f(\cdot)$ of the FL:

$$
\sum_{t=1}^{T_{1}}\left[g\left(R V_{t}\right)-g\left(\sigma_{t}^{2}\right)\right]^{2}
$$

where $t=1, \ldots, T_{1}$ is the IS period and $g(\cdot)$ is a monotonic function. Choosing $g(\cdot)$ from the $f$-menu of identity, square-root and logarithmic functions, leads to LS, SDLS and LNLS estimation criteria, respectively. Since they allow for the simple OLS estimation of the models' parameters, LS is the estimation criterion of choice for the HAR of Corsi (2009) and LNLS for the LOG - HAR of Andersen et al. (2007). Do notice 
that $\sigma_{t}^{2}$ in the EC is that defined by the forecasting loss: $\sigma_{t}^{2}=f^{-1}\left(\mathbb{E}_{t-1}\left[f\left(R V_{t}\right)\right]\right)$. Therefore, unless $g=f$, there is a misalignment between what the model parameters are trained to predict, $\mathbb{E}_{t-1}\left[g\left(R V_{t}\right)\right]$, and the object of interest $\mathbb{E}_{t-1}\left[f\left(R V_{t}\right)\right]$.

\section{$2.2 \quad$ Kullback-Leibler Distance}

Of the many density functions that may characterize a Kullback-Leibler distance we focus on the Gaussian density to generate the QML forecasting loss:

$$
\sum_{t=T_{1}+1}^{T_{2}}\left[\ln \sigma_{t}^{2}+\frac{R V_{t}}{\sigma_{t}^{2}}\right]
$$

where $t=T_{1}+1, \ldots, T_{2}$ is the OOS period and $\sigma_{t}^{2}$ is the conditional expectation $\mathbb{E}_{t-1}\left[R V_{t}\right]$ of realized variance. Similarly, the QML estimation criterion is given by:

$$
\sum_{t=1}^{T_{1}}\left[\ln \sigma_{t}^{2}+\frac{R V_{t}}{\sigma_{t}^{2}}\right]
$$

where $t=1, \ldots, T_{1}$ is the IS period and $\sigma_{t}^{2}=f^{-1}\left(\mathbb{E}_{t-1}\left[f\left(R V_{t}\right)\right]\right)$, as defined by the FL. Since the Gaussian belongs to the family of linear-exponential distributions, minimization of the QML estimation criterion is in fact a quasi-maximum-likelihood estimation with associated properties: Gourièroux et al. (1984). If intraday observations are normally distributed, Gaussian QML is maximum likelihood. Although differently motivated, the EC in equation (1) is the estimator of choice for the univariate MEM of Brownlees et al. (2012).

\section{$3 \quad$ Variance Modeling}

In this section we present the cores of the HAR and several other models inspired by successful GARCH parameterizations, which nest various specifications that have been put forward in the literature on realized variance modeling. Motivated by the stylized facts of GARCH modeling, we focus on the $(1,1)$ and $(2,1)$ parameterizations where the former is found to be well suited to generate good forecasts, as highlighted by Hansen and Lunde (2005), while the latter occasionally provided better fit and forecasts.

We present all models as parameterizing $\sigma_{t}^{2}$. Although this is non-standard, it is consistent with our setup in which the model parameters are chosen IS to generate the best predictions $\mathbb{E}_{t-1}\left[g\left(\sigma_{t}^{2}\right)\right]$ but are ultimately used to produce OOS forecasts 
$\mathbb{E}_{t-1}\left[f\left(\sigma_{t}^{2}\right)\right]$. While our empirical analysis focuses on one-step ahead forecasts, $k$-stepahead forecasts $\mathbb{E}_{t-1}\left[f\left(\sigma_{t+k-1}^{2}\right)\right]$ may be generated for any of the following parameterizations, granting that in general their calculation requires numerical integration for $k>1$.

\subsection{HAR Specifications}

The HAR, introduced by Corsi (2009), has rapidly achieved the status of benchmark specification for the modeling of realized variances. Features that contribute to its role as a point of reference are the simplicity with which its parameters may be estimated and its ability to reproduce long memory features: "[...] the mixing of relatively few volatility components is capable of reproducing a remarkably slow volatility autocorrelation decay that is almost indistinguishable from that of a hyperbolic pattern over most empirically relevant forecast horizons" (Andersen et al., 2007). The HAR models $\sigma_{t}^{2}$ as a function of past realizations over daily, weekly and monthly time intervals:

$$
\sigma_{t}^{2}=\omega+\alpha_{1} \cdot R V_{t-1}+\alpha_{2} \cdot \frac{1}{5} \sum_{i=1}^{5} R V_{t-i}+\alpha_{3} \cdot \frac{1}{22} \sum_{i=1}^{22} R V_{t-i}
$$

corresponding to an $A R(22)$ process for $R V_{t}$ with parameter constraints. Its parameters may be estimated by ordinary least squares when the criterion used in estimation is LS, whereas for different choices of the EC the estimates are not available in closed form. Necessary and sufficient conditions for the positivity of $\sigma_{t}^{2}$ are $\omega>0, \alpha_{3} \geqslant 0$, $\alpha_{2} / 5+\alpha_{3} / 22 \geqslant 0$ and $\alpha_{1}+\alpha_{2} / 5+\alpha_{3} / 22 \geqslant 0$.

The LOG - HAR, introduced by Andersen et al. (2007), is an alternative specification linear in the logarithms:

$$
\sigma_{t}^{2}=\exp \left\{\omega+\alpha_{1} \cdot \ln R V_{t-1}+\alpha_{2} \cdot \ln \left(\frac{1}{5} \sum_{i=1}^{5} R V_{t-i}\right)+\alpha_{3} \cdot \ln \left(\frac{1}{22} \sum_{i=1}^{22} R V_{t-i}\right)\right\}
$$

Notice that the presence of the logarithms of averages places the LOG - HAR outside the class of AR processes. Its parameters may be estimated by ordinary least squares for LNLS estimation criterion, while for different choices of the EC the estimates are not available in closed form.

\subsection{MVAR Specification}

With MVAR we indicate the parameterization of $\sigma_{t}^{2}$ in terms of its lags and lags of $R V_{t}$ or, equivalently, the ARMA modeling of realized variance $R V_{t}$. It is inspired 
by the $\operatorname{GARCH}(p, q)$ of Bollerslev (1986) from which it may be obtained by substituting the more precise realized variances for the squared open-to-close returns. This GARCH would arise as the limiting case of MVAR when the number of intradaily observations is one and realized variance collapses to the squared open-to-close return. The $\operatorname{MVAR}(2,1)$ specification is given by:

$$
\sigma_{t}^{2}=\omega+\alpha_{1} \cdot R V_{t-1}+\alpha_{2} \cdot R V_{t-2}+\beta_{1} \cdot \sigma_{t-1}^{2}
$$

Necessary and sufficient conditions for the positivity of $\sigma_{t}^{2}$ are $\omega>0, \alpha_{1}, \beta_{1} \geqslant 0$ and $\alpha_{2} \geqslant-\alpha_{1} \beta_{1}$. MVAR estimated by QML coincides with the MEM of Cipollini et al. (2013) while associated to the LS criterion it reduces to standard ARMA modeling and estimation.

\subsection{MVOL Specification}

With MVOL we denote the the parameterization of $\sigma_{t}$ in terms of its lags and lags of $R V_{t}^{1 / 2}$ or, equivalently, the ARMA modeling of realized volatility $R V_{t}^{1 / 2}$. It is related to the $\operatorname{TGARCH}(p, q)$ of Zakoian (1994), but without the asymmetric term, from which it may be obtained by substituting realized volatilities for the absolute value of the returns. Alternatively, the TGARCH may be seen as the limiting case of MVOL when there is one intradaily observation and realized volatility collapses to the absolute open-to-close return. The symmetric $\operatorname{MVOL}(2,1)$ specification is given by:

$$
\sigma_{t}^{2}=\left\{\omega+\alpha_{1} \cdot R V_{t-1}^{1 / 2}+\alpha_{2} \cdot R V_{t-2}^{1 / 2}+\beta_{1} \cdot \sigma_{t-1}\right\}^{2}
$$

Although $\sigma_{t}^{2}$ is positive by construction, the marginal effects of its determinants do not exhibit abrupt sign changes if and only if $\sigma_{t}$ is also positive. Necessary and sufficient conditions for the positivity of $\sigma_{t}$ are $\omega>0, \alpha_{1}, \beta_{1} \geqslant 0$ and $\alpha_{2} \geqslant-\alpha_{1} \beta_{1}$. QML estimation of MVOL coincides with the MEM in Brownlees et al. (2012) while adopting the SDLS criterion reduces to standard ARMA modeling and estimation.

\subsection{MLOG Specification}

MLOG is the ARMA modeling of the log realized variances $\ln R V_{t}$ or, equivalently, the parameterization of $\ln \sigma_{t}^{2}$ in terms of its lags and lags of $\ln R V_{t}$. It may be seen as the analogue to the $\log \operatorname{GARCH}(p, q)$ of Geweke (1986) from which it may be obtained by substituting log realized variances for log squared residuals. Similarly, log-GARCH is the limiting case of MLOG when the log realized variance reduces to 
$\log$ squared residual for a single intradaily observation. The $\operatorname{MLOG}(2,1)$ specification is given by:

$$
\sigma_{t}^{2}=\exp \left\{\omega+\alpha_{1} \cdot \ln R V_{t-1}+\alpha_{2} \cdot \ln R V_{t-2}+\beta_{1} \cdot \ln \sigma_{t-1}^{2}\right\}
$$

When associated to the LNLS criterion it reduces to standard ARMA modeling and estimation. QML, on the other hand, would be the natural estimator within the MEM framework. Dynamic specifications analogous to the MLOG are not uncommon in the context of Autoregressive Conditional Durations, among which Bauwens et al. (2008) and Taylor and Xu (2017) are examples of $\mathrm{QML}^{1}$ and LNLS estimates, respectively.

\subsection{MEXP Specification}

Inspired by the $\operatorname{EGARCH}(p, q)$ parameterization of Nelson (1991), but without the asymmetric term, the $\operatorname{MEXP}(2,1)$ specification is obtained by substituting realized volatilities for the absolute value of the return. Like for the other GARCH-inspired parameterizations, in the limiting case of a single intraday observation, MEXP reproduces the symmetric EGARCH:

$$
\sigma_{t}^{2}=\exp \left\{\omega+\alpha_{1} \cdot \frac{R V_{t-1}^{1 / 2}}{\sigma_{t-1}}+\alpha_{2} \cdot \frac{R V_{t-2}^{1 / 2}}{\sigma_{t-2}}+\beta_{1} \cdot \ln \sigma_{t-1}^{2}\right\}
$$

Notice how the presence of lags of the dependent variable at the denominator places MEXP outside the class of ARMA processes.

\section{Model Evaluation}

We perform IS model evaluation and selection by means of the Bayes (Schwarz) Information Criterion BIC. For the quadratic estimation criteria of Section 2.1 we construct the $B I C$ by treating the generalized residuals $g\left(R V_{t}\right)-g\left(\sigma_{t}^{2}\right)$ as Gaussian:

$$
B I C=T_{1} \ln \left(\frac{1}{T_{1}} \sum_{t=1}^{T_{1}}\left[g\left(R V_{t}\right)-g\left(\hat{\sigma}_{t}^{2}\right)\right]^{2}\right)+k \ln T_{1}
$$

where $k$ is the number of parameters, $T_{1}$ the sample size and $\hat{\sigma}_{t}^{2}$ the model's prediction. For the Kullback-Leibler estimation criterion of Section 2.2, the BIC is immediately

\footnotetext{
${ }^{1}$ To be precise: Bauwens et al. (2008) estimate an ACD analogous to MLOG by minimizing the Kullback-Leibler distance based on the exponential distribution. Since the exponential belongs to the family of linear-exponential distributions, the resulting estimator is also quasi-maximum-likelihood.
} 
obtained from the log-likelihood function:

$$
B I C=\sum_{t=1}^{T_{1}} \ln \hat{\sigma}_{t}^{2}+k \ln T_{1}
$$

where $\hat{\sigma}_{t}^{2}$ is the prediction from the QML-estimated model. Notice how the $B I C$ is calculated on the condensed estimation criterion in (1) from which the average of $R V_{t} / \sigma_{t}^{2}$ is dropped on the grounds that deviations from its limiting value of one are neither data nor model driven but only reflect initial value choices ${ }^{2}$.

Since, for equally parameterized specifications, every information criterion produces identical model rankings, Akaike's and Hannan-Quinn's may produce results that differ from those we present only in the comparisons of differently parameterized specifications. Furthermore, given that $B I C$ is the most conservative of the three, when it selects a richer parameterization so do Akaike's and Hannan-Quinn's.

We evaluate OOS forecasts directly from the forecasting loss functions of Sections 2.1 and 2.2. The reason why no penalties are needed is that, contrary to IS, OOS measures of fit are not non-decreasing functions of the number of parameters $k$.

\section{Data}

The data used in this study pertains to 28 of the 30 constituents of the Dow Jones 30 Index. The sample has 11 years of high-frequency daily observations from 01/03/2005 to $12 / 31 / 2015$ for a total of 2768 days. The two series, with tickers TRV and $\mathrm{V}$, are not included in the study because they are not available for the full sample period $^{3}$. Tickers of the 28 included stocks are: AAPL, AXP , BA, CAT, CSCO, CVX, DD, DIS, GE, GS, HD, IBM, INTC, JNJ, JPM, KO, MCD, MMM, MRK, MSFT, NKE, PFE, PG, UNH, UTX, VZ, WMT, XOM. The raw tick-by-tick TAQ data is cleaned using the procedure of Brownlees and Gallo (2006) and the series of realized variances calculated following Barndorff-Nielsen et al. (2011) with Parzen kernel. For every stock the time series of realized variances have been Winsorized at the 99.9th percentile (3 observations) to eliminate the possibly disturbing effects of spurious measures such as those recorded on the day of the Flash Crash of May 6, 2010.

The sample is split into six 5-year IS periods: 2005-2009 (1259 obs.), 2006-2010 (1259 obs.), 2007-2011 (1260 obs.), 2008-2012 (1259 obs.), 2009-2013 (1258 obs.) and

\footnotetext{
${ }^{2}$ In fact, for all the specifications considered, when the initial value $\sigma_{0}^{2}$ is treated as an unknown parameter and estimated, the average ratio $R V_{t} / \sigma_{t}^{2}$ is equal to one.

${ }^{3}$ TRV data are available only from $02 / 26 / 2007$ while $\mathrm{V}$ data are missing from $08 / 04 / 2006$ to $02 / 26 / 2007$.
} 
2010-2014 (1258 obs.). All model combinations are estimated on each of the six IS periods, and for each of them, OOS forecasts are generated for the following 1-year period: 2010 (252 obs.), 2011 (252 obs.), 2012 (250 obs.), 2013 (252 obs.), 2014 (252 obs.) and 2015 (251 obs.).

\section{Results}

The first result we document is the poor performance of the MEXP specification inspired by the successful EGARCH: Tables 9-12. It never provides the best IS description of the data ${ }^{4}$ and it always generates the largest OOS forecasting losses: $300 \%$ and $50 \%$ increments with respect to LS and SDLS, respectively. Perhaps this is $\mathrm{due}^{5}$ to the different nature of the shocks driving the processes: return shocks in the case of the EGARCH against variance shocks in the MEXP. Due to these findings we do not discuss the the MEXP specification any further in what follows.

\subsection{Is the forecasting loss the best estimation criterion?}

In Tables 1-8 we evaluate the conditional variance specifications (rows) for a given FL when estimated by LS, SDLS, LNLS and QML estimation criteria (columns). From Tables 1 and 2 it emerges that when the FL is LS or SDLS, the estimation criterion that produces the best OOS results in most instances is LNLS. When the FL is LNLS, Table 3 highlights that it should also be used as EC as it produces the best OOS results in $86.90 \%$ to $94.64 \%$ of the instances. On the other hand, when the FL is QML, Table 4 shows that overall QML is the preferred EC.

These results are confirmed in Tables 5-8 which, for every conditional variance specification (rows) and estimation criterion (columns), report the average value of the given FL over the OOS period and across assets. The lowest average OOS forecasting loss measured by LS, SDLS and LNLS are obtained, for every variance specification, when estimated using the LNLS estimation criterion. Similarly, the QML estimation criterion produces the lowest OOS QML forecasting losses. Hence, while the hypothesis that the functional form of FL provides the best EC has to be rejected as a general statement, it cannot be rejected for the QML forecasting loss.

\footnotetext{
${ }^{4}$ The only exception is observed for the LS forecasting loss for which $\operatorname{MEXP}(2,1)$ is the best IS specification $1.79 \%$ of the instances, Table 9 .

${ }^{5}$ While another possible explanation may be related to invertibility issues (see Wintenberger (2013)), in our case it does not seems to be the driving force: focusing on one step-ahead forecasts strongly mitigates any ill behavior (even explosive processes do not explode) and imposing invertibility constraints will further reduce the already poor IS performance of MEXP.
} 


\subsection{Does BIC identify the best OOS specification?}

From Tables 9-12: BIC selects the best OOS specification 26.00\%, 30.00\%, 38.00\% and $3.00 \%$ of the instances for LS, SDLS, LNLS and QML estimation criteria, respectively. Hence, it clearly emerges that the ability of BIC to identify what will be the best OOS specification is rather hopeless when associated to the QML estimation criterion. This finding is a bit surprising since the BIC associated to the QML estimation criterion follows directly from the EC itself as opposed to the other cases where BIC is constructed by treating the generalized residuals, from the various measures of distance, as normally distributed. In Tables 9 and 12 the average OOS forecasting loss of the specifications selected by BIC is no smaller than that of any of the specifications considered. On the other hand, the average forecasting loss of the BIC selection for the SDLS and LNLS forecasting loss functions is the second smallest, preceded only by the $\operatorname{MLOG}(2,1)$ : Tables 10 and 11 .

Therefore, for big caps, the best OOS results are obtained by adopting the MLOG specification for every asset. Clearly, it would be a hasty generalization to extend such recommendation to medium and small caps or other asset classes. A judicious approach would suggest to rely on model selection procedures unless the superiority of a specific model for a given asset class is substantiated by empirical evidence. Similarly, the performance of a maintained model should be monitored over time and asset classes to detect significant changes and intervene when necessary.

\subsection{Is there a best OOS specification?}

Tables 9-12 provide further IS and OOS performance measures for when the FL functional is used as EC. To begin, HAR does not emerge as the best OOS specification for any of the FL considered. Specifically, HAR is the best OOS specification $13.69 \%$, $8.93 \%, 10.71 \%$ and $21.43 \%$ of the instances for LS, SDLS, LNLS and QML, respectively. Focusing on the average OOS forecasting losses generated by HAR it emerges that its performance is in-between that of $\operatorname{MVAR}(1,1)$ and $\operatorname{MVAR}(2,1)$, with all other specifications exhibiting lower OOS forecasting losses.

LOG - HAR produces the best OOS fit $16.07 \%, 8.33 \%, 10.71 \%$ and $6.55 \%$ of the instances for LS, SDLS, LNLS and QML forecasting losses, respectively. Similarly to HAR, it too does not emerge as the preferred OOS specification. In terms of average OOS forecasting losses, LOG - HAR performs better than HAR and MVAR but does not do as well as $\operatorname{MVOL}(2,1)$ and MLOG. 
Considering HAR and LOG - HAR jointly doesn't alter the picture as they provide the best OOS fit $29.76 \%, 17.26 \%, 21.42 \%$ and $27.98 \%$ of the instances for LS, SDLS, LNLS and QML forecasting losses, respectively. In contrast, MLOG produces the best OOS specifications $47.02 \%, 64.88 \%, 64.88 \%$ and $54.16 \%$ of the times. In fact, what emerges is that the specification producing the smallest average OOS values of the FL considered is $\operatorname{MLOG}(2,1)$ followed nearly ex aequo by $\operatorname{MLOG}(1,1)$ and $\operatorname{MVOL}(2,1)$.

\subsection{Is there a best IS specification?}

Even though the main focus of this study is on OOS forecasts, we recognize that there are instances where IS fit is of primary importance, e.g., a reduced form model capturing most relevant data features to be used as auxiliary model in the indirect estimation of a maintained model. In Tables 9-12, HAR does not emerge as the best IS specification: it provides the best IS fit only $4.76 \%, 4.17 \%, 2.38 \%$ and $12.50 \%$ of the instances for LS, SDLS, LNLS and QML estimation criteria, respectively.

LOG - HAR provides the best IS fit $13.10 \%, 30.95 \%, 25.00 \%$ and $24.40 \%$ of the instances for LS, SDLS, LNLS and QML estimation criteria, respectively. While there are sizeable improvements with respect to HAR, LOG - HAR is the preferred specification (30.95\% of the instances) only for the QML estimation criterion with $\operatorname{MLOG}(1,1)$ a very close second (30.36\% of the times). On the other hand, with respect to the other EC considered, MLOG is selected from two to five more times than LOG - HAR as best IS specification.

Jointly, HAR and LOG - HAR are the preferred specifications $29.76 \%, 28.57 \%$, $15.48 \%$ and $43.45 \%$ of the instances for LS, SDLS, LNLS and QML estimation criteria, respectively. However, the corresponding performance of MLOG of $65.48 \%, 58.93 \%$, $70.24 \%$ and $30.36 \%$ is substantially better (except for QML). Furthermore, if the HAR and LOG - HAR pair is compared to the MLOG and MVOL pair, the latter is found to provide the best IS for any EC considered.

\section{General Discussion}

\subsection{On the Estimation Criteria}

A possible reading key to the empirical findings promoting LNLS and QML as preferred EC is that of their sensitivity to what we call relatively-extreme observations to distinguish them from outliers. Outliers are those data points that are distant 
from the rest of the observations, according to some measure. In our context, outliers correspond to extreme measures of $R V_{t}$ which, in general, are relatively easy to detect and to deal with ${ }^{6}$. Instead, by relatively-extreme observations we mean those data points that, while not being intrinsically anomalous, are extreme with respect to certain data features, e.g., with $R V_{t-1}$ and $R V_{t+1}$ in the 5-th percentile of magnitudes, for an otherwise persistent process, an $R V_{t}$ in the 90-th percentile constitutes a relatively-extreme observation but not an outlier in the usual sense.

Focusing on the fact that parameters' estimates respond to relatively-extreme observations by re-setting the estimators' first order conditions to zero, we elicit the following two insights ${ }^{7}$. First, the impact of a relatively-extreme observation on the generalized residual, $\hat{\varepsilon}_{t}=g\left(R V_{t}\right)-g\left(\hat{\sigma}_{t}^{2}\right)$ for the criteria of Section 2.1 and $\hat{\varepsilon}_{t}=R V_{t}-\widehat{\sigma}_{t}^{2}$ for the criterion of Section 2.2, is smallest for LNLS followed by SDLS and the pair of equals LS and QML. Secondly, the contribution of a relatively-extreme observation to the first order conditions is proportional to $\widehat{\sigma}_{t}^{-n}$ where $\widehat{\sigma}_{t}^{2}$ is the model's prediction, and $n=\{0,1,2,4\}$ for LS, SDLS, LNLS and QML, respectively. It follows that inverse powers of $\widehat{\sigma}_{t}^{2}$ have a dampening effect on the generalized residuals which is strongest for QML, followed by LNLS, SDLS and LS. Hence, SDLS and LS emerge as more sensitive to relatively-extreme observations in line with the empirical findings which don't promote any of the two as preferred EC.

\subsection{The $(2,1)$ Parameterizations}

The positive performance of MLOG and MVOL may be ascribed, among others, to the inclusion of the components associated with the coefficients $\beta_{1}$ and $\alpha_{2}$. The relevance of the moving-average coefficient $\beta_{1}$ should not come as a surprise given its ubiquitous presence in GARCH models. The $(2,1)$ parameterizations of MVAR, MVOL and MLOG are found to produce better forecasts than their $(1,1)$ counterparts: Tables 5-8. Figure 1 reports the distributions of the $(2,1)$ specifications' parameters estimated by LNLS and QML. The striking empirical regularity is the negative sign of the $\widehat{\alpha}_{2}$ coefficients for every specification, asset and sub-sample period. As shown by Figure 2, the effect of $\alpha_{2}<0$ is to reduce (increase) persistence at short (long) horizons with respect to the geometrically decaying correlations of the $(1,1)$ parameterizations. Analogously, the negative relation summarized by $\alpha_{2}<0$ is not captured by the core

\footnotetext{
${ }^{6}$ In our study, the 99.9-th percentile Winsorization of the data effectively removes the few outliers present such as that associated to the Flash Crash of May 6, 2010.

${ }^{7}$ For a detailed treatment of the robustness of M-estimators, see Hampel et al. (2005).
} 
HAR specifications.

\subsection{Long Memory Approximation}

Among the features that have made the HAR specifications popular is their ability to approximate long memory processes and thus replicate the hyperbolic decay observed in the autocorrelation of realized variances. The cross-sectional average (over 28 assets) of the empirical autocorrelations of realized variances (DATA), for the most recent subsample (2010-2014), is plotted in Figure 2. Using the cross-sectional average of the parameters' estimates for the same subsample, $10^{6}$ Monte Carlo simulations, from the $\operatorname{MVAR}(2,1), \operatorname{MVOL}(2,1), \operatorname{MLOG}(2,1), \operatorname{HAR}$ and LOG - HAR, are used to plot the models' autocorrelation functions in Figure 2. The decay of DATA autocorrelations from lag-12 is well approximated by all specifications with the exception of the LOG - HAR, which reproduces the correct rate of decay but under-estimates the magnitude of the autocorrelations. Most interestingly, all $(2,1)$ specifications have autocorrelation structures that are indistinguishable from the HAR. Hence, replication of long-range dependence is not an exclusive feature of the HAR family but one attainable by equally parsimonious specifications. Equally interesting are the results for the autocorrelations up to lag-11. At lag-1, we find autocorrelations that are approximately 0.6 for the DATA, 0.67 and 0.5 for the HAR and LOG - HAR, respectively, but only $0.3-0.4$ for the $(2,1)$ specifications.

These findings may be reconciled with the observed superior forecasts of MLOG by realizing that replicating the autocorrelation function of the observables is not a sufficient condition for a model to produce quality forecasts. Consider the following counter-example in which $R V_{t}$ and two competing forecasts $\hat{\sigma}_{1, t}^{2}$ and $\hat{\sigma}_{2, t}^{2}$ are given by:

$$
\begin{aligned}
R V_{t} & =\sigma_{t}^{2}+\nu_{t} \\
\hat{\sigma}_{1, t}^{2} & =\sigma_{t}^{2}+S_{1} \eta_{1, t} \\
\hat{\sigma}_{2, t}^{2} & =\sigma_{t}^{2}+S_{2} \eta_{2, t}
\end{aligned}
$$

with $1<S_{2}<S_{1} ; \nu_{t}$ and $\eta_{2, t}$ i.i.d. standardized random variables; $\sigma_{t}^{2}$ a unit variance process with lag- $j$ autocovariance $\gamma_{j} ; \eta_{1, t}$ a standardized random variable with lag- $j$ autocovariance $\psi_{j}=0.5 S_{1}^{-2}\left(S_{1}^{2}-1\right) \gamma_{j}$. Then, the autocorrelations of the three processes are $\rho_{j}(R V)=0.5 \gamma_{j}, \rho_{j}\left(\hat{\sigma}_{1}^{2}\right)=0.5 \gamma_{j}$ and $\rho_{j}\left(\hat{\sigma}_{2}^{2}\right)=\left(1+S_{2}^{2}\right)^{-1} \gamma_{j}$, respectively. The forecasting mean square errors of the competing specifications are: $\mathbb{E}\left[\left(R V_{t}-\hat{\sigma}_{1, t}^{2}\right)^{2}\right]=\mathbb{E}\left[\left(R V_{t}-\sigma_{t}^{2}\right)^{2}\right]+S_{1}^{2}$ and $\mathbb{E}\left[\left(R V_{t}-\hat{\sigma}_{2, t}^{2}\right)^{2}\right]=\mathbb{E}\left[\left(R V_{t}-\sigma_{t}^{2}\right)^{2}\right]+S_{2}^{2}$. Therefore, for $1<S_{2}<S_{1}$, the autocorrelation structure of $\hat{\sigma}_{1, t}^{2}$ is an exact match of 
that of $R V_{t}$ while $\hat{\sigma}_{2, t}^{2}$ exhibits lower autocorrelations. Nevertheless, the forecasting mean square error of $\hat{\sigma}_{2, t}^{2}$ is lower than that of $\hat{\sigma}_{1, t}^{2}$. It is therefore possible to have alternative specifications producing more precise forecasts than HAR even though the lag-1 autocorrelation of HAR is substantially higher as a result of larger misspecification components.

This analysis is supported by the contemporaneous correlations between realized variances and the models' forecasts: 0.6153 for the $\operatorname{MVAR}(2,1), 0.6219$ for the $\operatorname{MVOL}(2,1), 0.6231$ for the $\operatorname{MLOG}(2,1), 0.5026$ for the HAR and 0.6174 for the LOG - HAR. Hence, the similarity of the HAR correlation structure with that of realized variance is not necessarily indicative of a good specification. With respect to the latter, consider the limiting case of two independent processes $y_{t}$ and $x_{t}$ with identical model specifications and parameter values. By construction, the autocorrelation function of $x_{t}$ is a perfect match for that of $y_{t}$. However, because the two processes are independent $x_{t}, \mathbb{E}_{t-1}\left[x_{t}\right]$ and $x_{t-1}$ are worst forecasters of $y_{t}$ than $\bar{y}$, which, with i.i.d. innovations, has zero autocorrelations. Therefore, while the autocorrelation structure of the true model matches that of the data, matching that structure is not a sufficient condition for the identification of a good model.

\section{Conclusions}

In this paper we advance the idea that out-of-sample forecast evaluation relies on the choice of a distance between predicted outcomes and actual values of realized variance (as well as volatility and log-variance) that reflects subjective preferences. Conditional on a specific OOS forecasting loss, we show that the same functional form may not be the most appropriate choice as an IS estimation criterion in which distance between actual and fitted values is used to deliver parameter estimates. To this end, we have examined several models inspired by well known GARCH parameterizations alongside HAR specifications and we have handled several combinations of IS estimation criteria and OOS forecasting losses.

We find the $(2,1)$ parameterizations to be best suited to model the dynamics and forecast realized variances, volatilities and log-variances. In particular, in terms of OOS forecasts: $\operatorname{MVAR}(2,1)$ does generally better than $\operatorname{HAR} ; \operatorname{MVOL}(2,1)$ does generally better than LOG - HAR; MLOG does always better than LOG - HAR. Interestingly, our empirical findings point to the presence of a negative lag-2 coefficient in all estimated $(2,1)$ specifications. We interpret it as a dampening agent which, by 
limiting to one day most of the impact of a shock to variance, induces a mimicking effect of long memory properties that are indistinguishable from those of the HAR and more sustained than those of the LOG - HAR.

With respect to the estimators considered, we find that for all quadratic forecasting losses, models estimated using the LNLS estimation criterion provide the best OOS forecasts. On the other hand, for the QML forecasting loss the QML estimation criterion does better than LNLS, although marginally so. Our findings further suggest a judicious approach to model selection which should rely on information criteria unless the superiority of a specific model is substantiated by empirical evidence.

We leave the evaluation of models combining the elements of the HAR family with those of a $(p, q)$ parameterization to future research.

\section{References}

Andersen, T. G., Bollerslev, T. and Diebold, F. X. (2007) Roughing it up: Including jump components in the measurement, modeling and forecasting of return volatility, Review of Economics and Statistics, 89, 701-720.

Barndorff-Nielsen, O. E., Hansen, P. R., Lunde, A. and Shephard, N. (2008) Designing realised kernels to measure the ex-post variation of equity prices in the presence of noise, Econometrica, 76, 1481-1536.

Barndorff-Nielsen, O. E., Hansen, P. R., Lunde, A. and Shephard, N. (2011) Multivariate realised kernels: Consistent positive semi-definite estimators of the covariation of equity prices with noise and non-synchronous trading, Journal of Econometrics, 162, 149-169.

Bauwens, L., Galli, F. and Giot, P. (2008) The moments of log-ACD models, Quantitative and Qualitative Analysis in Social Sciences, 2, 1-28.

Bollerslev, T. (1986) Generalized autoregressive conditional heteroskedasticity, Journal of Econometrics, 31, 307-327.

Bollerslev, T., Patton, A. J. and Quaedvlieg, R. (2016) Exploiting the errors: A simple approach for improved volatility forecasting, Journal of Econometrics, 192, $1-18$. 
Brownlees, C. T., Cipollini, F. and Gallo, G. M. (2012) Multiplicative error models, in Volatility Models and Their Applications (Eds.) L. Bauwens, C. Hafner and S. Laurent, Wiley, pp. 223-247.

Cipollini, F., Engle, R. F. and Gallo, G. M. (2013) Semiparametric vector MEM, Journal of Applied Econometrics, 28, 1067-1086.

Corsi, F. (2009) A simple approximate long-memory model of realized volatility, Journal of Financial Econometrics, 7, 174-196.

Engle, R. F. and Gallo, G. M. (2006) A multiple indicators model for volatility using intra-daily data, Journal of Econometrics, 131, 3-27.

Geweke, J. (1986) Modeling the persistence in conditional variances: A comment, Econometric Reviews, 5, 57-61.

Gourièroux, C., Monfort, A. and Trognon, A. (1984) Pseudo maximum likelihood methods: Theory, Econometrica, 52, 681-700.

Hampel, F., Ronchetti, E., Rousseeuw, P. and Stahel, W. (2005) Robust Statistics: The Approach Based on Influence Functions, Wiley, New York, 2-nd edn.

Nelson, D. B. (1991) Conditional heteroskedasticity in asset returns: A new approach, Econometrica, 59, 347-370.

Park, S. and Linton, O. (2012) Realized volatility: Theory and applications, in Volatility Models and Their Applications (Eds.) L. Bauwens, C. Hafner and S. Laurent, Wiley, pp. 319-345.

Taylor, N. and Xu, Y. (2017) The logarithmic vector multiplicative error model: an application to high frequency NYSE stock data, Quantitative Finance, 17, 10211035.

Wintenberger, O. (2013) Continuous invertibility and stable QML estimation of the EGARCH(1,1), Scandinavian Journal of Statistics, 40, 846-867.

Zakoian, J.-M. (1994) Threshold heteroskedastic models, Journal of Economic Dynamics and Control, 18, 931 - 955. 


\section{Table 1:}

LS forecasting loss for out-of-sample evaluations: for each of the four estimation criteria (LS, SDLS, LNLS and QML), in each row we report the number of instances (and the percentages) when that criterion provides the best performance (by row 28 tickers times 6 periods $=168$ possible instances). The $\chi_{3}^{2}$ column reports $p$-values under the null hypothesis of a uniform best performance across the four estimation criteria.

\begin{tabular}{|c|c|c|c|c|c|c|c|c|c|c|}
\hline \multicolumn{2}{|l|}{ Model } & \multicolumn{2}{|r|}{$\mathrm{LS}$} & \multicolumn{2}{|c|}{ SDLS } & \multicolumn{2}{|c|}{ LNLS } & \multicolumn{2}{|c|}{ QML } & \multirow{2}{*}{$\frac{\chi_{3}^{2}}{<0.0001}$} \\
\hline MVAR & $(1,1)$ & 18 & $10.71 \%$ & 23 & $13.69 \%$ & 115 & $68.45 \%$ & 12 & $7.14 \%$ & \\
\hline & $(2,1)$ & 22 & $13.10 \%$ & 32 & $19.05 \%$ & 98 & $58.33 \%$ & 16 & $9.52 \%$ & $<0.0001$ \\
\hline \multirow[t]{2}{*}{ MEXP } & $(1,1)$ & 20 & $11.90 \%$ & 20 & $11.90 \%$ & 99 & $58.93 \%$ & 29 & $17.26 \%$ & $<0.0001$ \\
\hline & $(2,1)$ & 35 & $20.83 \%$ & 29 & $17.26 \%$ & 45 & $26.79 \%$ & 59 & $35.12 \%$ & 0.0071 \\
\hline \multirow[t]{2}{*}{ MVOL } & $(1,1)$ & 13 & $7.74 \%$ & 30 & $17.86 \%$ & 113 & $67.26 \%$ & 12 & $7.14 \%$ & $<0.0001$ \\
\hline & $(2,1)$ & 18 & $10.71 \%$ & 36 & $21.43 \%$ & 92 & $54.76 \%$ & 22 & $13.10 \%$ & $<0.0001$ \\
\hline \multirow[t]{2}{*}{ MLOG } & $(1,1)$ & 14 & $8.33 \%$ & 43 & $25.60 \%$ & 89 & $52.98 \%$ & 22 & $13.10 \%$ & $<0.0001$ \\
\hline & $(2,1)$ & 21 & $12.50 \%$ & 46 & $27.38 \%$ & 66 & $39.29 \%$ & 35 & $20.83 \%$ & $<0.0001$ \\
\hline \multicolumn{2}{|l|}{ HAR } & 19 & $11.31 \%$ & 23 & $13.69 \%$ & 103 & $61.31 \%$ & 23 & $13.69 \%$ & $<0.0001$ \\
\hline \multicolumn{2}{|c|}{ LOG-HAR } & 11 & $6.55 \%$ & 40 & $23.81 \%$ & 93 & $55.36 \%$ & 24 & $14.29 \%$ & $<0.0001$ \\
\hline
\end{tabular}

\section{Table 2:}

SDLS forecasting loss function for out-of-sample evaluations: for each of the four estimation criteria (LS, SDLS, LNLS and QML), in each row we report the number of instances (and the percentages) when that criterion provides the best performance (by row 28 tickers times 6 periods $=168$ possible instances). The $\chi_{3}^{2}$ column reports $p$-values under the null hypothesis of a uniform best performance across the four estimation criteria.

\begin{tabular}{|c|c|c|c|c|c|c|c|c|c|c|}
\hline \multicolumn{2}{|l|}{ Model } & \multicolumn{2}{|c|}{ LS } & \multicolumn{2}{|c|}{ SDLS } & \multicolumn{2}{|c|}{ LNLS } & \multicolumn{2}{|c|}{ QML } & \multirow{2}{*}{$\begin{array}{r}\chi_{3}^{2} \\
<0.0001\end{array}$} \\
\hline MVAR & $(1,1)$ & 14 & $8.33 \%$ & 19 & $11.31 \%$ & 134 & $79.76 \%$ & 1 & $0.60 \%$ & \\
\hline & $(2,1)$ & 6 & $3.57 \%$ & 40 & $23.81 \%$ & 122 & $72.62 \%$ & 0 & $0.00 \%$ & $<0.0001$ \\
\hline \multirow[t]{2}{*}{ MEXP } & $(1,1)$ & 12 & $7.14 \%$ & 12 & $7.14 \%$ & 131 & $77.98 \%$ & 13 & $7.74 \%$ & $<0.0001$ \\
\hline & $(2,1)$ & 18 & $10.71 \%$ & 30 & $17.86 \%$ & 75 & $44.64 \%$ & 45 & $26.79 \%$ & $<0.0001$ \\
\hline \multirow[t]{2}{*}{ MVOL } & $(1,1)$ & 4 & $2.38 \%$ & 31 & $18.45 \%$ & 130 & $77.38 \%$ & 3 & $1.79 \%$ & $<0.0001$ \\
\hline & $(2,1)$ & 2 & $1.19 \%$ & 46 & $27.38 \%$ & 118 & $70.24 \%$ & 2 & $1.19 \%$ & $<0.0001$ \\
\hline \multirow[t]{2}{*}{ MLOG } & $(1,1)$ & 2 & $1.19 \%$ & 49 & $29.17 \%$ & 116 & $69.05 \%$ & 1 & $0.60 \%$ & $<0.0001$ \\
\hline & $(2,1)$ & 5 & $2.98 \%$ & 61 & $36.31 \%$ & 99 & $58.93 \%$ & 3 & $1.79 \%$ & $<0.0001$ \\
\hline \multicolumn{2}{|l|}{ HAR } & 5 & $2.98 \%$ & 30 & $17.86 \%$ & 132 & $78.57 \%$ & 1 & $0.60 \%$ & $<0.0001$ \\
\hline \multicolumn{2}{|c|}{ LOG-HAR } & 3 & $1.79 \%$ & 45 & $26.79 \%$ & 117 & $69.64 \%$ & 3 & $1.79 \%$ & $<0.0001$ \\
\hline
\end{tabular}




\section{Table 3:}

LNLS forecasting loss for out-of-sample evaluations: for each of the four estimation criteria (LS, SDLS, LNLS and $\mathrm{QML}$ ), in each row we report the number of instances (and the percentages) when that criterion provides the best performance (by row 28 tickers times 6 periods $=168$ possible instances). The $\chi_{3}^{2}$ column reports $p$-values under the null hypothesis of a uniform best performance across the four estimation criteria.

\begin{tabular}{|cc|ccccccccc|}
\hline \hline Model & & \multicolumn{2}{c}{ LS } & \multicolumn{2}{c}{ SDLS } & \multicolumn{2}{c}{ LNLS } & \multicolumn{2}{c}{ QML } & $\chi_{3}^{2}$ \\
\hline \hline \multirow{2}{*}{ MVAR } & $(1,1)$ & 3 & $1.79 \%$ & 19 & $11.31 \%$ & 146 & $86.90 \%$ & 0 & $0.00 \%$ & $<0.0001$ \\
& $(2,1)$ & 1 & $0.60 \%$ & 13 & $7.74 \%$ & 154 & $91.67 \%$ & 0 & $0.00 \%$ & $<0.0001$ \\
MEXP & $(1,1)$ & 0 & $0.00 \%$ & 10 & $5.95 \%$ & 157 & $93.45 \%$ & 1 & $0.60 \%$ & $<0.0001$ \\
& $(2,1)$ & 3 & $1.79 \%$ & 24 & $14.29 \%$ & 122 & $72.62 \%$ & 19 & $11.31 \%$ & $<0.0001$ \\
\multirow{2}{*}{ MVOL } & $(1,1)$ & 1 & $0.60 \%$ & 18 & $10.71 \%$ & 149 & $88.69 \%$ & 0 & $0.00 \%$ & $<0.0001$ \\
& $(2,1)$ & 0 & $0.00 \%$ & 9 & $5.36 \%$ & 159 & $94.64 \%$ & 0 & $0.00 \%$ & $<0.0001$ \\
\multirow{2}{*}{ MLOG } & $(1,1)$ & 0 & $0.00 \%$ & 17 & $10.12 \%$ & 151 & $89.88 \%$ & 0 & $0.00 \%$ & $<0.0001$ \\
& $(2,1)$ & 0 & $0.00 \%$ & 18 & $10.71 \%$ & 150 & $89.29 \%$ & 0 & $0.00 \%$ & $<0.0001$ \\
\multirow{2}{*}{ HAR } & & 0 & $0.00 \%$ & 15 & $8.93 \%$ & 153 & $91.07 \%$ & 0 & $0.00 \%$ & $<0.0001$ \\
\multicolumn{2}{|c}{ LOG-HAR } & 0 & $0.00 \%$ & 15 & $8.93 \%$ & 153 & $91.07 \%$ & 0 & $0.00 \%$ & $<0.0001$ \\
\hline \hline
\end{tabular}

\section{Table 4:}

QML forecasting loss for out-of-sample evaluations: for each of the four estimation criteria (LS, SDLS, LNLS and $\mathrm{QML}$ ), in each row we report the number of instances (and the percentages) when that criterion provides the best performance (by row 28 tickers times 6 periods $=168$ possible instances). The $\chi_{3}^{2}$ column reports $p$-values under the null hypothesis of a uniform best performance across the four estimation criteria.

\begin{tabular}{|cc|ccccccccc|}
\hline \hline Model & & \multicolumn{2}{c}{ LS } & \multicolumn{2}{c}{ SDLS } & \multicolumn{2}{c}{ LNLS } & \multicolumn{2}{c}{ QML } & $\chi_{3}^{2}$ \\
\hline \hline \multirow{2}{*}{ MVAR } & $(1,1)$ & 23 & $13.69 \%$ & 45 & $26.79 \%$ & 48 & $28.57 \%$ & 52 & $30.95 \%$ & 0.0036 \\
& $(2,1)$ & 27 & $16.07 \%$ & 38 & $22.62 \%$ & 32 & $19.05 \%$ & 71 & $42.26 \%$ & $<0.0001$ \\
MEXP & $(1,1)$ & 18 & $10.71 \%$ & 32 & $19.05 \%$ & 32 & $19.05 \%$ & 86 & $51.19 \%$ & $<0.0001$ \\
& $(2,1)$ & 16 & $9.52 \%$ & 26 & $15.48 \%$ & 17 & $10.12 \%$ & 109 & $64.88 \%$ & $<0.0001$ \\
MVOL & $(1,1)$ & 33 & $19.64 \%$ & 49 & $29.17 \%$ & 35 & $20.83 \%$ & 51 & $30.36 \%$ & 0.1008 \\
& $(2,1)$ & 39 & $23.21 \%$ & 36 & $21.43 \%$ & 23 & $13.69 \%$ & 70 & $41.67 \%$ & $<0.0001$ \\
MLOG & $(1,1)$ & 39 & $23.21 \%$ & 38 & $22.62 \%$ & 34 & $20.24 \%$ & 57 & $33.93 \%$ & 0.0700 \\
& $(2,1)$ & 49 & $29.17 \%$ & 27 & $16.07 \%$ & 26 & $15.48 \%$ & 66 & $39.29 \%$ & $<0.0001$ \\
\multirow{2}{*}{ HAR } & & 25 & $14.88 \%$ & 31 & $18.45 \%$ & 34 & $20.24 \%$ & 78 & $46.43 \%$ & $<0.0001$ \\
\multicolumn{2}{|c}{ LOG-HAR } & 49 & $29.17 \%$ & 24 & $14.29 \%$ & 23 & $13.69 \%$ & 72 & $42.86 \%$ & $<0.0001$ \\
\hline \hline
\end{tabular}




\section{Table 5:}

LS forecasting loss for out-of-sample evaluations: for each of the four estimation criteria (LS, SDLS, LNLS and QML), in each row we report the average loss (by row 28 tickers times 6 periods $=168$ possible instances). For every specification, the lowest loss (across estimation criteria) is reported in bold. The $F_{3, \infty}$ column reports $p$-values under the null hypothesis of a uniform average performance across the four estimation criteria.

\begin{tabular}{|cc|rrrrr|}
\hline \hline Model & & LS & SDLS & LNLS & QML & $F_{3, \infty}$ \\
\hline \hline \multirow{2}{*}{ MVAR } & $(1,1)$ & 3.2112 & 3.1890 & $\mathbf{3 . 1 5 3 7}$ & 3.3789 & $<0.0001$ \\
& $(2,1)$ & 3.1254 & 3.1060 & $\mathbf{3 . 1 0 0 8}$ & 3.2990 & $<0.0001$ \\
MEXP & $(1,1)$ & $\mathbf{1 3 . 0 1 2 4}$ & 58.6417 & 35.2893 & 70.0017 & 0.3240 \\
& $(2,1)$ & $\mathbf{1 2 . 7 7 7 7}$ & 81.0910 & 78.6324 & 159.0144 & 0.2989 \\
MVOL & $(1,1)$ & 3.0772 & 2.9607 & $\mathbf{2 . 9 1 5 0}$ & 3.0102 & $<0.0001$ \\
& $(2,1)$ & 3.0108 & 2.9170 & $\mathbf{2 . 8 8 8 3}$ & 2.9610 & $<0.0001$ \\
MLOG & $(1,1)$ & 2.9830 & 2.8480 & $\mathbf{2 . 8 4 0 7}$ & 2.8517 & $<0.0001$ \\
& $(2,1)$ & 2.9613 & 2.8201 & 2.8241 & $\mathbf{2 . 8 1 2 0}$ & $<0.0001$ \\
\multirow{2}{*}{ HAR } & & 3.1432 & 3.1256 & $\mathbf{3 . 1 0 8 5}$ & 3.3150 & $<0.0001$ \\
\multicolumn{2}{|c|}{ LOG-HAR } & 3.0755 & 2.9114 & $\mathbf{2 . 8 8 2 8}$ & 2.9347 & $<0.0001$ \\
\hline \hline
\end{tabular}

\section{Table 6:}

SDLS forecasting loss for out-of-sample evaluations: for each of the four estimation criteria (LS, SDLS, LNLS and QML), in each row we report the average loss (by row 28 tickers times 6 periods $=168$ possible instances). For every specification, the lowest loss (across estimation criteria) is reported in bold. The $F_{3, \infty}$ column reports $p$-values under the null hypothesis of a uniform average performance across the four estimation criteria.

\begin{tabular}{|cc|rrrrr|}
\hline \hline Model & & LS & SDLS & LNLS & QML & $F_{3, \infty}$ \\
\hline \hline \multirow{2}{*}{ MVAR } & $(1,1)$ & 0.1164 & 0.1045 & $\mathbf{0 . 1 0 2 9}$ & 0.1096 & $<0.0001$ \\
& $(2,1)$ & 0.1082 & 0.1003 & $\mathbf{0 . 0 9 9 3}$ & 0.1055 & $<0.0001$ \\
MEXP & $(1,1)$ & $\mathbf{0 . 1 5 0 3}$ & 0.1629 & 0.1552 & 0.2012 & 0.1121 \\
& $(2,1)$ & $\mathbf{0 . 1 5 5 6}$ & 0.1710 & 0.1727 & 0.2170 & 0.1778 \\
MVOL & $(1,1)$ & 0.1128 & 0.0992 & $\mathbf{0 . 0 9 7 5}$ & 0.1027 & $<0.0001$ \\
& $(2,1)$ & 0.1056 & 0.0961 & $\mathbf{0 . 0 9 5 0}$ & 0.0994 & $<0.0001$ \\
MLOG & $(1,1)$ & 0.1084 & 0.0961 & $\mathbf{0 . 0 9 5 0}$ & 0.0986 & $<0.0001$ \\
& $(2,1)$ & 0.1055 & 0.0939 & $\mathbf{0 . 0 9 3 3}$ & 0.0960 & $<0.0001$ \\
\multirow{2}{*}{ HAR } & & 0.1082 & 0.1009 & $\mathbf{0 . 0 9 9 7}$ & 0.1057 & $<0.0001$ \\
LOG-HAR & 0.1077 & 0.0968 & $\mathbf{0 . 0 9 5 6}$ & 0.0997 & $<0.0001$ \\
\hline \hline
\end{tabular}




\section{Table 7:}

LNLS forecasting loss for out-of-sample evaluations: for each of the four estimation criteria (LS, SDLS, LNLS and QML), in each row we report the average loss (by row 28 tickers times 6 periods=168 possible instances). For every specification, the lowest loss (across estimation criteria) is reported in bold. The $F_{3, \infty}$ column reports $p$-values under the null hypothesis of a uniform average performance across the four estimation criteria.

\begin{tabular}{|cc|ccccc|}
\hline \hline Model & & LS & SDLS & LNLS & QML & $F_{3, \infty}$ \\
\hline \hline MVAR & $(1,1)$ & 0.2471 & 0.1955 & $\mathbf{0 . 1 8 8 1}$ & 0.2074 & $<0.0001$ \\
& $(2,1)$ & 0.2215 & 0.1880 & $\mathbf{0 . 1 8 2 4}$ & 0.2012 & $<0.0001$ \\
MEXP & $(1,1)$ & 0.2521 & 0.2042 & $\mathbf{0 . 1 9 6 1}$ & 0.2170 & $<0.0001$ \\
& $(2,1)$ & 0.2613 & 0.2154 & $\mathbf{0 . 2 0 7 5}$ & 0.2204 & $<0.0001$ \\
MVOL & $(1,1)$ & 0.2351 & 0.1885 & $\mathbf{0 . 1 8 2 7}$ & 0.2004 & $<0.0001$ \\
& $(2,1)$ & 0.2145 & 0.1820 & $\mathbf{0 . 1 7 7 6}$ & 0.1943 & $<0.0001$ \\
MLOG & $(1,1)$ & 0.2205 & 0.1847 & $\mathbf{0 . 1 7 9 9}$ & 0.1954 & $<0.0001$ \\
& $(2,1)$ & 0.2114 & 0.1796 & $\mathbf{0 . 1 7 5 6}$ & 0.1903 & $<0.0001$ \\
HAR & & 0.2194 & 0.1885 & $\mathbf{0 . 1 8 2 5}$ & 0.2009 & $<0.0001$ \\
LOG-HAR & 0.2113 & 0.1826 & $\mathbf{0 . 1 7 8 6}$ & 0.1944 & $<0.0001$ \\
\hline \hline
\end{tabular}

\section{Table 8:}

QML forecasting loss for out-of-sample evaluations: for each of the four estimation criteria (LS, SDLS, LNLS and QML), in each row we report the average loss (by row 28 tickers times 6 periods $=168$ possible instances). For every specification, the lowest loss (across estimation criteria) is reported in bold. The $F_{3, \infty}$ column reports $p$-values under the null hypothesis of a uniform average performance across the four estimation criteria.

\begin{tabular}{|cc|rcccc|}
\hline \hline Model & & LS & SDLS & LNLS & QML & $F_{3, \infty}$ \\
\hline \hline MVAR & $(1,1)$ & 1.1297 & 1.1184 & 1.1206 & $\mathbf{1 . 1 1 8 1}$ & $<0.0001$ \\
& $(2,1)$ & 1.1236 & 1.1177 & 1.1199 & $\mathbf{1 . 1 1 6 6}$ & $<0.0001$ \\
MEXP & $(1,1)$ & 1.1323 & 1.1244 & 1.1257 & $\mathbf{1 . 1 2 1 8}$ & $<0.0001$ \\
& $(2,1)$ & 1.1362 & 1.1283 & 1.1290 & $\mathbf{1 . 1 2 3 3}$ & $<0.0001$ \\
MVOL & $(1,1)$ & 1.1246 & 1.1167 & 1.1199 & $\mathbf{1 . 1 1 6 5}$ & $<0.0001$ \\
& $(2,1)$ & 1.1198 & 1.1163 & 1.1193 & $\mathbf{1 . 1 1 5 2}$ & $<0.0001$ \\
MLOG & $(1,1)$ & 1.1200 & 1.1164 & 1.1201 & $\mathbf{1 . 1 1 5 7}$ & $<0.0001$ \\
& $(2,1)$ & 1.1184 & 1.1160 & 1.1196 & $\mathbf{1 . 1 1 4 7}$ & $<0.0001$ \\
\multirow{2}{*}{ HAR } & & 1.1230 & 1.1179 & 1.1200 & $\mathbf{1 . 1 1 6 6}$ & $<0.0001$ \\
\multicolumn{2}{|c|}{ LOG-HAR } & 1.1191 & 1.1173 & 1.1207 & $\mathbf{1 . 1 1 5 9}$ & $<0.0001$ \\
\hline \hline
\end{tabular}




\section{Table 9:}

LS forecasting loss and estimation criterion. The first three columns report the percentage a specification is best: IS, in-sample (based on BIC); OOS, out-of-sample (LS loss); OOS|IS, best out-of-sample, limiting to the best in-sample. A-Loss and M-Loss are the average and median losses. The BIC row contains out-of-sample results for the best specifications selected in-sample by the information criterion. Individual A-Losses are tested against the corresponding value on the bottom row $(\mathrm{a} * * *, * * *$ signifies a better performance $-10 \%, 5 \%, 1 \%$ significance the reverse for the corresponding $*, * *, * * *)$.

\begin{tabular}{|cc|ccccc|}
\hline \hline Model & & IS & OOS & OOS|IS & A-Loss & M-Loss \\
\hline \hline \multirow{2}{*}{ MVAR } & $(1,1)$ & $0.00 \%$ & $0.00 \%$ & $0.00 \%$ & 3.2112 & 1.1267 \\
& $(2,1)$ & $0.00 \%$ & $16.67 \%$ & $0.00 \%$ & 3.1254 & 1.0458 \\
MEXP & $(1,1)$ & $0.00 \%$ & $0.60 \%$ & $0.00 \%$ & 13.0124 & 1.1750 \\
& $(2,1)$ & $1.79 \%$ & $1.79 \%$ & $0.00 \%$ & 12.7777 & 1.2316 \\
MVOL & $(1,1)$ & $0.00 \%$ & $0.00 \%$ & $0.00 \%$ & 3.0772 & 1.1160 \\
& $(2,1)$ & $2.98 \%$ & $4.17 \%$ & $0.00 \%$ & 3.0108 & 1.0674 \\
MLOG & $(1,1)$ & $31.55 \%$ & $10.12 \%$ & $18.87 \%$ & 2.9830 & 1.0741 \\
& $(2,1)$ & $33.93 \%$ & $36.90 \%$ & $38.60 \%$ & 2.9613 & 1.0544 \\
HAR & & $4.76 \%$ & $13.69 \%$ & $0.00 \%$ & 3.1432 & 1.0221 \\
LOG-HAR & $25.00 \%$ & $16.07 \%$ & $30.95 \%$ & 3.0755 & 1.0705 \\
\hline BIC & & & $26.00 \%$ & & 3.2400 & 1.0731 \\
\hline \hline
\end{tabular}

\section{Table 10:}

SDLS forecasting loss and estimation criterion. The first three columns report the percentage a specification is best: IS, in-sample (based on $B I C$ ); OOS, out-of-sample (SDLS loss); OOS|IS, best out-of-sample, limiting to the best in-sample. A-Loss and M-Loss are the average and median losses. The BIC row contains out-of-sample results for the best specifications selected in-sample by the information criterion. Individual A-Losses are tested against the corresponding value on the bottom row $(\mathrm{a} * * *, * * *$ signifies a better performance $-10 \%, 5 \%, 1 \%$ significance - the reverse for the corresponding $*, * *, * * *)$.

\begin{tabular}{|cc|ccccc|}
\hline \hline Model & & IS & OOS & OOS|IS & A-Loss & M-Loss \\
\hline \hline MVAR & $(1,1)$ & $0.00 \%$ & $0.00 \%$ & $0.00 \%$ & $0.1045_{* * *}$ & 0.0770 \\
& $(2,1)$ & $0.60 \%$ & $7.74 \%$ & $0.00 \%$ & $0.1003_{* * *}$ & 0.0756 \\
MEXP & $(1,1)$ & $0.00 \%$ & $2.38 \%$ & $0.00 \%$ & $0.1629_{*}$ & 0.0818 \\
& $(2,1)$ & $0.00 \%$ & $0.60 \%$ & $0.00 \%$ & $0.1710_{*}$ & 0.0880 \\
MVOL & $(1,1)$ & $0.00 \%$ & $0.00 \%$ & $0.00 \%$ & $0.0992_{* * *}$ & 0.0753 \\
& $(2,1)$ & $11.90 \%$ & $7.14 \%$ & $20.00 \%$ & 0.0961 & 0.0743 \\
MLOG & $(1,1)$ & $7.74 \%$ & $10.12 \%$ & $15.38 \%$ & 0.0961 & 0.0748 \\
& $(2,1)$ & $51.19 \%$ & $54.76 \%$ & $47.67 \%$ & $0.0939_{* * *}$ & 0.0736 \\
HAR & & $4.17 \%$ & $8.93 \%$ & $0.00 \%$ & $0.1009_{* * *}$ & 0.0751 \\
LOG-HAR & $24.40 \%$ & $8.33 \%$ & $9.76 \%$ & $0.0968_{*}$ & 0.0744 \\
\hline \multicolumn{2}{|c|}{ BIC } & & $30.00 \%$ & & 0.0959 & 0.0736 \\
\hline \hline
\end{tabular}




\section{Table 11:}

LNLS forecasting loss and estimation criterion. The first three columns report the percentage a specification is best: IS, in-sample (based on $B I C$ ); OOS, out-of-sample (LNLS loss); OOS|IS, best out-of-sample, limiting to the best in-sample. A-Loss and M-Loss are the average and median losses. The BIC row contains out-of-sample results for the best specifications selected in-sample by the information criterion. Individual A-Losses are tested against the corresponding value on the bottom row $(\mathrm{a} * * *, * * *$ signifies a better performance $-10 \%, 5 \%, 1 \%$ significance - the reverse for the corresponding $*, * *, * * *)$.

\begin{tabular}{|cc|ccccc|}
\hline \hline Model & & IS & OOS & OOS|IS & A-Loss & M-Loss \\
\hline \hline \multirow{2}{*}{ MVAR } & $(1,1)$ & $0.00 \%$ & $0.00 \%$ & $0.00 \%$ & $0.1881_{* * *}$ & 0.1809 \\
& $(2,1)$ & $0.00 \%$ & $5.36 \%$ & $0.00 \%$ & $0.1824_{* * *}$ & 0.1759 \\
MEXP & $(1,1)$ & $0.00 \%$ & $0.00 \%$ & $0.00 \%$ & $0.1961_{* * *}$ & 0.1808 \\
& $(2,1)$ & $0.00 \%$ & $1.19 \%$ & $0.00 \%$ & $0.2075_{* * *}$ & 0.1966 \\
MVOL & $(1,1)$ & $0.00 \%$ & $0.60 \%$ & $0.00 \%$ & $0.1827_{* * *}$ & 0.1770 \\
& $(2,1)$ & $14.29 \%$ & $6.55 \%$ & $8.33 \%$ & $0.1776_{* * *}$ & 0.1724 \\
MLOG & $(1,1)$ & $1.79 \%$ & $10.12 \%$ & $0.00 \%$ & $0.1799_{* * *}$ & 0.1741 \\
& $(2,1)$ & $68.45 \%$ & $54.76 \%$ & $51.30 \%$ & $0.1756^{* * *}$ & 0.1699 \\
HAR & & $2.38 \%$ & $10.71 \%$ & $0.00 \%$ & $0.1825_{* * *}$ & 0.1754 \\
LOG-HAR & $13.10 \%$ & $10.71 \%$ & $18.18 \%$ & $0.1786_{* * *}$ & 0.1739 \\
\hline BIC & & & $38.00 \%$ & & 0.1765 & 0.1705 \\
\hline \hline
\end{tabular}

\section{Table 12:}

QML forecasting loss and estimation criterion. The first three columns report the percentage a specification is best: IS, in-sample (based on BIC); OOS, out-of-sample (QML loss); OOS|IS, best out-of-sample, limiting to the best in-sample. A-Loss and M-Loss are the average and median losses. The BIC row contains out-of-sample results for the best specifications selected in-sample by the information criterion. Individual A-Losses are tested against the corresponding value on the bottom row $(\mathrm{a} *, * *, * * *$ signifies a better performance $-10 \%, 5 \%, 1 \%$ significance the reverse for the corresponding $*, * *, * * *)$.

\begin{tabular}{|cc|ccccc|}
\hline \hline Model & & IS & OOS & OOS|IS & A-Loss & M-Loss \\
\hline \hline MVAR & $(1,1)$ & $8.33 \%$ & $2.38 \%$ & $0.00 \%$ & $1.1181_{* * *}$ & 1.0965 \\
& $(2,1)$ & $0.00 \%$ & $6.55 \%$ & $0.00 \%$ & 1.1166 & 1.0945 \\
MEXP & $(1,1)$ & $0.00 \%$ & $1.19 \%$ & $0.00 \%$ & $1.1218_{* * *}$ & 1.0991 \\
& $(2,1)$ & $0.00 \%$ & $1.19 \%$ & $0.00 \%$ & $1.1233_{* * *}$ & 1.0995 \\
MVOL & $(1,1)$ & $17.86 \%$ & $1.19 \%$ & $0.00 \%$ & 1.1165 & 1.0939 \\
& $(2,1)$ & $0.00 \%$ & $5.36 \%$ & $0.00 \%$ & $1.1152^{* * *}$ & 1.0917 \\
MLOG & $(1,1)$ & $30.36 \%$ & $17.26 \%$ & $3.92 \%$ & $1.1157^{* * *}$ & 1.0933 \\
& $(2,1)$ & $0.00 \%$ & $36.90 \%$ & $0.00 \%$ & $1.1147^{* * *}$ & 1.0908 \\
HAR & & $12.50 \%$ & $21.43 \%$ & $9.52 \%$ & 1.1166 & 1.0946 \\
LOG-HAR & $30.95 \%$ & $6.55 \%$ & $3.85 \%$ & $1.1159^{* *}$ & 1.0917 \\
\hline BIC & & $3.00 \%$ & & 1.1166 & 1.0951 \\
\hline \hline
\end{tabular}


Figure 1: Parameters' density estimates over the six IS periods and all the assets considered. From left to right, the columns contain the densities of the parameters $\alpha_{1}, \alpha_{2}$ and $\beta_{1}$ for the three specifications reported.
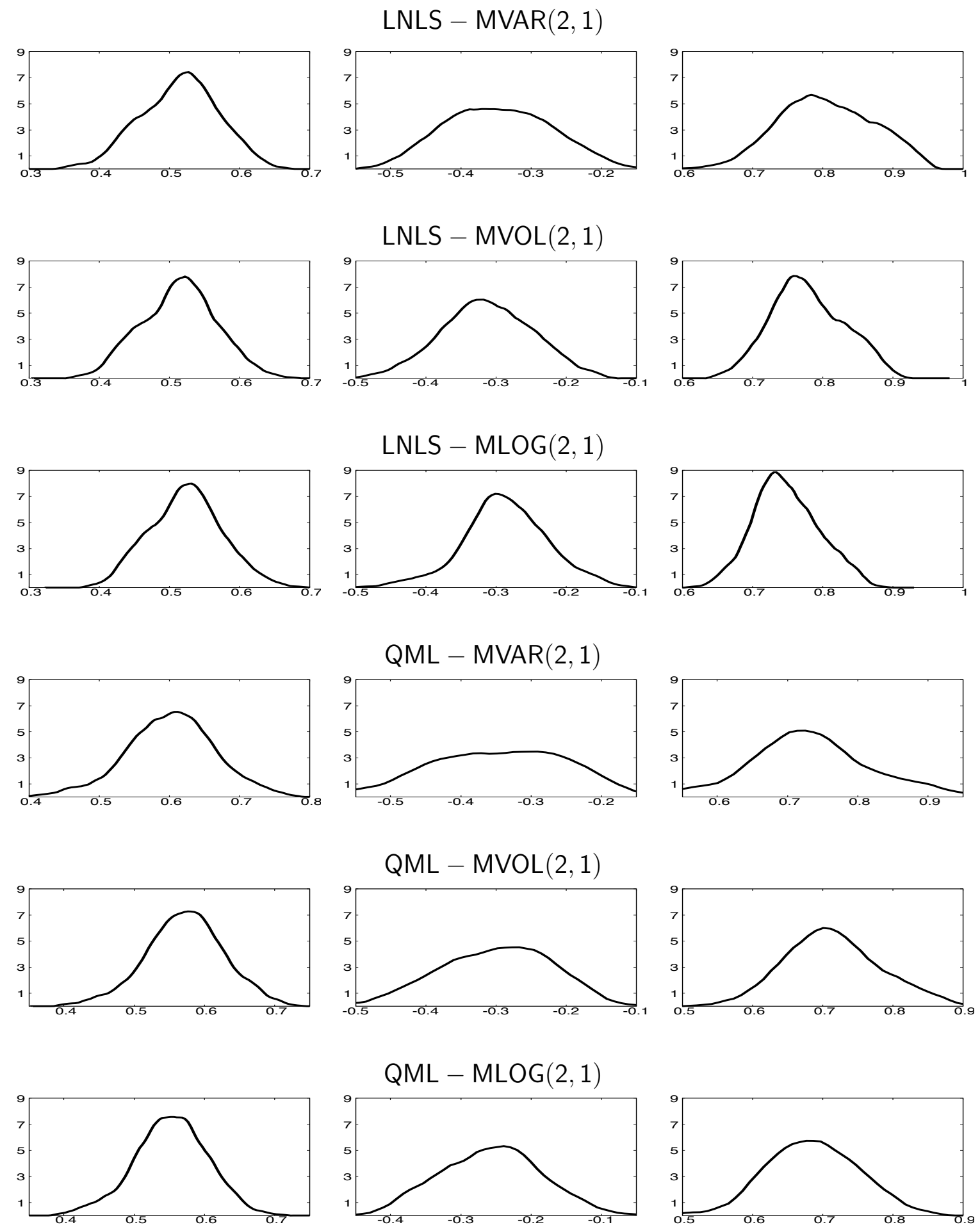
Figure 2: Autocorrelation functions for the variance processes with parameters equal to the cross-sectional averages of the LNLS estimates in the most recent subsample (20102014). MVAR, MVOL and MLOG specifications refer to the $(2,1)$ parameterizations. Autocorrelations are computed over $10^{6}$ simulations with additive standardized Gaussian innovations.

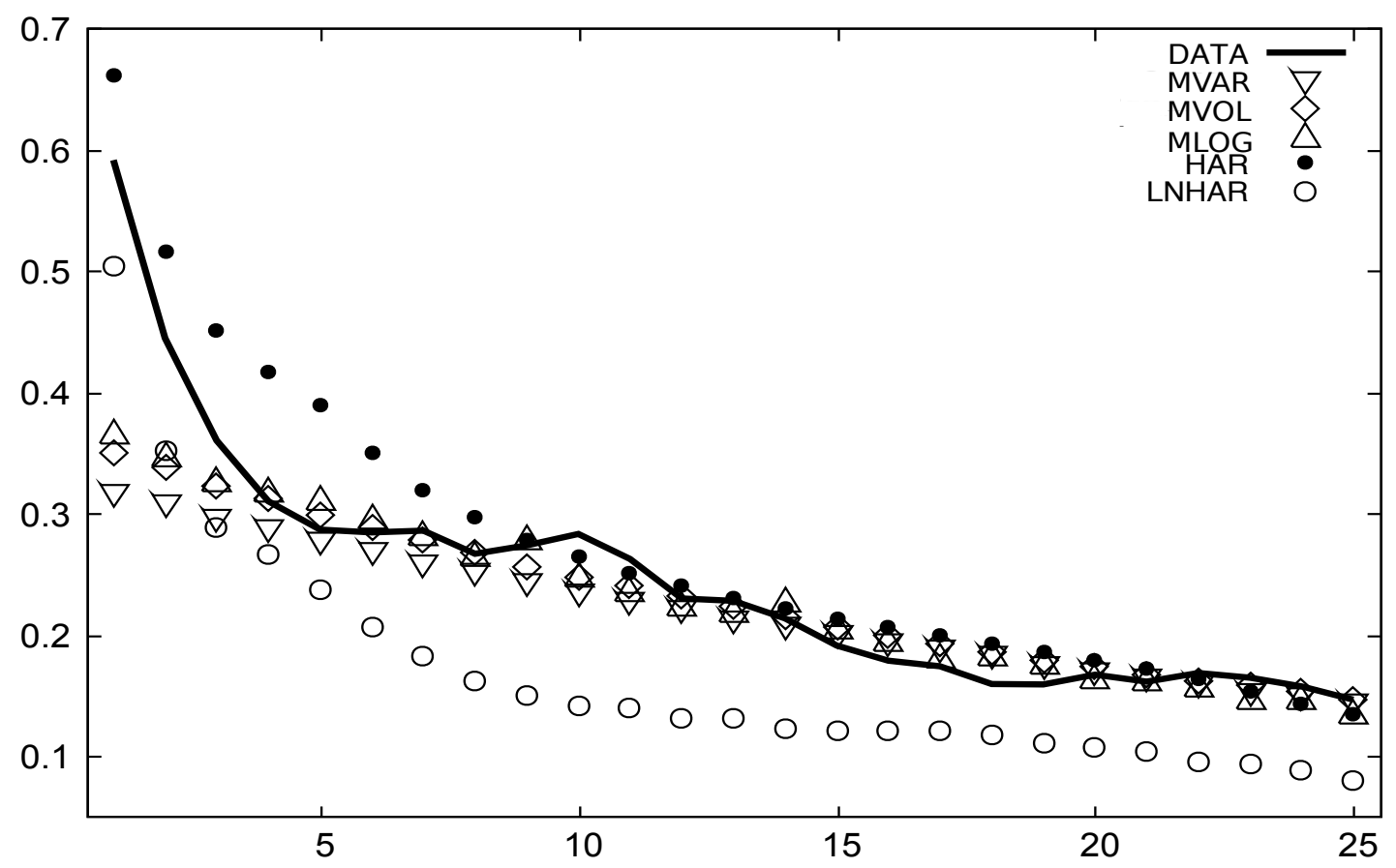




\section{Table 13:}

Tantamount to Table 1 but with no Winsorization of IS data. LS forecasting loss for out-of-sample evaluations: for each of the four estimation criteria (LS, SDLS, LNLS and QML), in each row we report the number of instances (and the percentages) when that criterion provides the best performance (by row 28 tickers times 6 periods $=168$ possible instances). The $\chi_{3}^{2}$ column reports $p$-values under the null hypothesis of a uniform best performance across the four estimation criteria.

\begin{tabular}{|c|c|c|c|c|c|c|c|c|c|c|}
\hline \multicolumn{2}{|l|}{ Model } & & \multicolumn{2}{|c|}{ SDLS } & \multicolumn{2}{|c|}{ LNLS } & \multicolumn{2}{|c|}{ QML } & \multirow{2}{*}{$\frac{\chi_{3}^{2}}{<0.0001}$} \\
\hline MVAR & $(1,1)$ & 26 & $15.48 \%$ & 34 & $20.24 \%$ & 95 & $56.55 \%$ & 13 & $7.74 \%$ & \\
\hline \multirow{3}{*}{ MEXP } & $(2,1)$ & 21 & $12.50 \%$ & 43 & $25.60 \%$ & 81 & $48.21 \%$ & 23 & $13.69 \%$ & $<0.0001$ \\
\hline & $(1,1)$ & 31 & $18.45 \%$ & 26 & $15.48 \%$ & 81 & $48.21 \%$ & 30 & $17.86 \%$ & $<0.0001$ \\
\hline & $(2,1)$ & 33 & $19.64 \%$ & 43 & $25.60 \%$ & 32 & $19.05 \%$ & 60 & $35.71 \%$ & 0.0092 \\
\hline \multirow[t]{2}{*}{ MVOL } & $(1,1)$ & 13 & $7.74 \%$ & 29 & $17.26 \%$ & 105 & $62.50 \%$ & 21 & $12.50 \%$ & $<0.0001$ \\
\hline & $(2,1)$ & 14 & $8.33 \%$ & 39 & $23.21 \%$ & 85 & $50.60 \%$ & 30 & $17.86 \%$ & $<0.0001$ \\
\hline \multirow[t]{2}{*}{ MLOG } & $(1,1)$ & 10 & $5.95 \%$ & 37 & $22.02 \%$ & 94 & $55.95 \%$ & 27 & $16.07 \%$ & $<0.0001$ \\
\hline & $(2,1)$ & 15 & $8.93 \%$ & 35 & $20.83 \%$ & 77 & $45.83 \%$ & 41 & $24.40 \%$ & $<0.0001$ \\
\hline \multicolumn{2}{|l|}{ HAR } & 24 & $14.29 \%$ & 39 & $23.21 \%$ & 78 & $46.43 \%$ & 27 & $16.07 \%$ & $<0.0001$ \\
\hline \multicolumn{2}{|c|}{ LOG-HAR } & 10 & $5.95 \%$ & 33 & $19.64 \%$ & 91 & $54.17 \%$ & 34 & $20.24 \%$ & $<0.0001$ \\
\hline
\end{tabular}

\section{Table 14:}

Tantamount to Table 2 but with no Winsorization of IS data. SDLS forecasting loss for out-of-sample evaluations: for each of the four estimation criteria (LS, SDLS, LNLS and QML), in each row we report the number of instances (and the percentages) when that criterion provides the best performance (by row 28 tickers times 6 periods $=168$ possible instances). The $\chi_{3}^{2}$ column reports $p$-values under the null hypothesis of a uniform best performance across the four estimation criteria.

\begin{tabular}{|c|c|c|c|c|c|c|c|c|c|c|}
\hline \multicolumn{2}{|l|}{ Model } & \multicolumn{2}{|c|}{$\mathrm{LS}$} & \multicolumn{2}{|c|}{ SDLS } & \multicolumn{2}{|c|}{ LNLS } & \multicolumn{2}{|c|}{ QML } & \multirow{2}{*}{$\frac{\chi_{3}^{2}}{2.0001}$} \\
\hline MVAR & $(1,1)$ & 13 & $7.74 \%$ & 23 & $13.69 \%$ & 131 & $77.98 \%$ & 1 & $0.60 \%$ & \\
\hline \multirow{3}{*}{ MEXP } & $(2,1)$ & 5 & $2.98 \%$ & 39 & $23.21 \%$ & 124 & $73.81 \%$ & 0 & $0.00 \%$ & $<0.0001$ \\
\hline & $(1,1)$ & 16 & $9.52 \%$ & 24 & $14.29 \%$ & 114 & $67.86 \%$ & 14 & $8.33 \%$ & $<0.0001$ \\
\hline & $(2,1)$ & 20 & $11.90 \%$ & 47 & $27.98 \%$ & 56 & $33.33 \%$ & 45 & $26.79 \%$ & 0.0002 \\
\hline \multirow[t]{2}{*}{ MVOL } & $(1,1)$ & 3 & $1.79 \%$ & 18 & $10.71 \%$ & 143 & $85.12 \%$ & 4 & $2.38 \%$ & $<0.0001$ \\
\hline & $(2,1)$ & 1 & $0.60 \%$ & 30 & $17.86 \%$ & 134 & $79.76 \%$ & 3 & $1.79 \%$ & $<0.0001$ \\
\hline \multirow[t]{2}{*}{ MLOG } & $(1,1)$ & 2 & $1.19 \%$ & 30 & $17.86 \%$ & 132 & $78.57 \%$ & 4 & $2.38 \%$ & $<0.0001$ \\
\hline & $(2,1)$ & 4 & $2.38 \%$ & 45 & $26.79 \%$ & 111 & $66.07 \%$ & 8 & $4.76 \%$ & $<0.0001$ \\
\hline \multicolumn{2}{|l|}{ HAR } & 4 & $2.38 \%$ & 37 & $22.02 \%$ & 126 & $75.00 \%$ & 1 & $0.60 \%$ & $<0.0001$ \\
\hline \multicolumn{2}{|c|}{ LOG-HAR } & 2 & $1.19 \%$ & 26 & $15.48 \%$ & 131 & $77.98 \%$ & 9 & $5.36 \%$ & $<0.0001$ \\
\hline
\end{tabular}




\section{Table 15:}

Tantamount to Table 3 but with no Winsorization of IS data. LNLS forecasting loss for out-of-sample evaluations: for each of the four estimation criteria (LS, SDLS, LNLS and QML), in each row we report the number of instances (and the percentages) when that criterion provides the best performance (by row 28 tickers times 6 periods $=168$ possible instances). The $\chi_{3}^{2}$ column reports $p$-values under the null hypothesis of a uniform best performance across the four estimation criteria.

\begin{tabular}{|c|c|c|c|c|c|c|c|c|c|c|}
\hline \multicolumn{2}{|l|}{ Model } & \multicolumn{2}{|c|}{$\mathrm{LS}$} & \multicolumn{2}{|c|}{ SDLS } & \multicolumn{2}{|c|}{ LNLS } & \multicolumn{2}{|c|}{ QML } & \multirow{2}{*}{$\frac{\chi_{3}^{2}}{<0.0001}$} \\
\hline MVAR & $(1,1)$ & 3 & $1.79 \%$ & 17 & $10.12 \%$ & 148 & $88.10 \%$ & 0 & $0.00 \%$ & \\
\hline \multirow{3}{*}{ MEXP } & $(2,1)$ & 1 & $0.60 \%$ & 12 & $7.14 \%$ & 155 & $92.26 \%$ & 0 & $0.00 \%$ & $<0.0001$ \\
\hline & $(1,1)$ & 0 & $0.00 \%$ & 15 & $8.93 \%$ & 152 & $90.48 \%$ & 1 & $0.60 \%$ & $<0.0001$ \\
\hline & $(2,1)$ & 3 & $1.79 \%$ & 28 & $16.67 \%$ & 118 & $70.24 \%$ & 19 & $11.31 \%$ & $<0.0001$ \\
\hline \multirow[t]{2}{*}{ MVOL } & $(1,1)$ & 1 & $0.60 \%$ & 16 & $9.52 \%$ & 151 & $89.88 \%$ & 0 & $0.00 \%$ & $<0.0001$ \\
\hline & $(2,1)$ & 0 & $0.00 \%$ & 8 & $4.76 \%$ & 160 & $95.24 \%$ & 0 & $0.00 \%$ & $<0.0001$ \\
\hline \multirow[t]{2}{*}{ MLOG } & $(1,1)$ & 0 & $0.00 \%$ & 14 & $8.33 \%$ & 154 & $91.67 \%$ & 0 & $0.00 \%$ & $<0.0001$ \\
\hline & $(2,1)$ & 0 & $0.00 \%$ & 16 & $9.52 \%$ & 152 & $90.48 \%$ & 0 & $0.00 \%$ & $<0.0001$ \\
\hline \multicolumn{2}{|l|}{ HAR } & 0 & $0.00 \%$ & 15 & $8.93 \%$ & 153 & $91.07 \%$ & 0 & $0.00 \%$ & $<0.0001$ \\
\hline \multicolumn{2}{|c|}{ LOG-HAR } & 0 & $0.00 \%$ & 12 & $7.14 \%$ & 156 & $92.86 \%$ & 0 & $0.00 \%$ & $<0.0001$ \\
\hline
\end{tabular}

\section{Table 16:}

Tantamount to Table 4 but with no Winsorization of IS data. QML forecasting loss for out-of-sample evaluations: for each of the four estimation criteria (LS, SDLS, LNLS and QML), in each row we report the number of instances (and the percentages) when that criterion provides the best performance (by row 28 tickers times 6 periods $=168$ possible instances). The $\chi_{3}^{2}$ column reports $p$-values under the null hypothesis of a uniform best performance across the four estimation criteria.

\begin{tabular}{|cc|cccccccccc}
\hline \hline Model & & \multicolumn{2}{c}{ LS } & SDLS & LNLS & QML & $\chi_{3}^{2}$ \\
\hline \hline MVAR & $(1,1)$ & 13 & $7.74 \%$ & 33 & $19.64 \%$ & 49 & $29.17 \%$ & 73 & $43.45 \%$ & $<0.0001$ \\
& $(2,1)$ & 16 & $9.52 \%$ & 28 & $16.67 \%$ & 39 & $23.21 \%$ & 85 & $50.60 \%$ & $<0.0001$ \\
MEXP & $(1,1)$ & 14 & $8.33 \%$ & 36 & $21.43 \%$ & 28 & $16.67 \%$ & 90 & $53.57 \%$ & $<0.0001$ \\
& $(2,1)$ & 11 & $6.55 \%$ & 29 & $17.26 \%$ & 19 & $11.31 \%$ & 109 & $64.88 \%$ & $<0.0001$ \\
MVOL & $(1,1)$ & 21 & $12.50 \%$ & 42 & $25.00 \%$ & 39 & $23.21 \%$ & 66 & $39.29 \%$ & $<0.0001$ \\
& $(2,1)$ & 25 & $14.88 \%$ & 34 & $20.24 \%$ & 30 & $17.86 \%$ & 79 & $47.02 \%$ & $<0.0001$ \\
MLOG & $(1,1)$ & 25 & $14.88 \%$ & 52 & $30.95 \%$ & 24 & $14.29 \%$ & 67 & $39.88 \%$ & $<0.0001$ \\
& $(2,1)$ & 30 & $17.86 \%$ & 44 & $26.19 \%$ & 22 & $13.10 \%$ & 72 & $42.86 \%$ & $<0.0001$ \\
HAR & & 13 & $7.74 \%$ & 28 & $16.67 \%$ & 39 & $23.21 \%$ & 88 & $52.38 \%$ & $<0.0001$ \\
LOG-HAR & 26 & $15.48 \%$ & 50 & $29.76 \%$ & 18 & $10.71 \%$ & 74 & $44.05 \%$ & $<0.0001$ \\
\hline \hline
\end{tabular}




\section{Table 17:}

Tantamount to Table 5 but with no Winsorization of IS data. LS forecasting loss for out-of-sample evaluations: for each of the four estimation criteria (LS, SDLS, LNLS and QML), in each row we report the average loss (by row 28 tickers times 6 periods $=168$ possible instances). For every specification, the lowest loss (across estimation criteria) is reported in bold. The $F_{3, \infty}$ column reports $p$-values under the null hypothesis of a uniform average performance across the four estimation criteria.

\begin{tabular}{|cc|rrrrr|}
\hline \hline Model & & LS & SDLS & LNLS & QML & \multicolumn{1}{c|}{$F_{3, \infty}$} \\
\hline \hline \multirow{2}{*}{ MVAR } & $(1,1)$ & 3.2963 & 3.1822 & $\mathbf{3 . 1 6 0 9}$ & 3.4345 & $<0.0001$ \\
& $(2,1)$ & 3.2135 & $\mathbf{3 . 1 0 4 6}$ & 3.1072 & 3.3609 & $<0.0001$ \\
MEXP & $(1,1)$ & $\mathbf{7 . 2 5 7 7}$ & 11.0253 & 32.8010 & 116.4256 & 0.0764 \\
& $(2,1)$ & $\mathbf{9 . 1 6 1 6}$ & 17.8734 & 70.8608 & 15825.0020 & 0.3728 \\
MVOL & $(1,1)$ & 3.2137 & 2.9635 & $\mathbf{2 . 9 1 6 6}$ & 3.0667 & $<0.0001$ \\
& $(2,1)$ & 3.1330 & 2.9307 & $\mathbf{2 . 8 9 0 9}$ & 3.0208 & $<0.0001$ \\
MLOG & $(1,1)$ & 3.1247 & 2.8597 & $\mathbf{2 . 8 4 2 3}$ & 2.9809 & 0.0020 \\
& $(2,1)$ & 3.1610 & 2.8436 & $\mathbf{2 . 8 2 5 9}$ & 2.9072 & $<0.0001$ \\
\multirow{2}{*}{ HAR } & & 3.2463 & 3.1192 & $\mathbf{3 . 1 1 4 1}$ & 3.3735 & $<0.0001$ \\
\multicolumn{2}{|c|}{ LOG-HAR } & 3.4370 & 2.9193 & $\mathbf{2 . 8 8 3 0}$ & 3.3062 & 0.0321 \\
\hline \hline
\end{tabular}

\section{Table 18:}

Tantamount to Table 6 but with no Winsorization of IS data. SDLS forecasting loss for out-of-sample evaluations: for each of the four estimation criteria (LS, SDLS, LNLS and QML), in each row we report the average loss (by row 28 tickers times 6 periods $=168$ possible instances). For every specification, the lowest loss (across estimation criteria) is reported in bold. The $F_{3, \infty}$ column reports $p$-values under the null hypothesis of a uniform average performance across the four estimation criteria.

\begin{tabular}{|cc|rcccc|}
\hline \hline Model & & LS & SDLS & LNLS & QML & $F_{3, \infty}$ \\
\hline \hline \multirow{2}{*}{ MVAR } & $(1,1)$ & 0.1342 & 0.1056 & $\mathbf{0 . 1 0 3 0}$ & 0.1110 & $<0.0001$ \\
& $(2,1)$ & 0.1246 & 0.1016 & $\mathbf{0 . 0 9 9 3}$ & 0.1073 & $<0.0001$ \\
MEXP & $(1,1)$ & 0.1547 & $\mathbf{0 . 1 3 1 6}$ & 0.1527 & 0.2303 & 0.0026 \\
& $(2,1)$ & 0.1686 & $\mathbf{0 . 1 4 0 8}$ & 0.1701 & 9.0428 & 0.3789 \\
MVOL & $(1,1)$ & 0.1293 & 0.0996 & $\mathbf{0 . 0 9 7 5}$ & 0.1042 & $<0.0001$ \\
& $(2,1)$ & 0.1211 & 0.0972 & $\mathbf{0 . 0 9 5 1}$ & 0.1013 & $<0.0001$ \\
MLOG & $(1,1)$ & 0.1209 & 0.0965 & $\mathbf{0 . 0 9 5 0}$ & 0.1009 & $<0.0001$ \\
& $(2,1)$ & 0.1222 & 0.0949 & $\mathbf{0 . 0 9 3 3}$ & 0.0984 & $<0.0001$ \\
\multirow{2}{*}{ HAR } & & 0.1256 & 0.1022 & $\mathbf{0 . 0 9 9 8}$ & 0.1073 & $<0.0001$ \\
\multicolumn{2}{|c|}{ LOG-HAR } & 0.1344 & 0.0974 & $\mathbf{0 . 0 9 5 5}$ & 0.1036 & $<0.0001$ \\
\hline \hline
\end{tabular}




\section{Table 19:}

Tantamount to Table 7 but with no Winsorization of IS data. LNLS forecasting loss for out-of-sample evaluations: for each of the four estimation criteria (LS, SDLS, LNLS and QML), in each row we report the average loss (by row 28 tickers times 6 periods $=168$ possible instances). For every specification, the lowest loss (across estimation criteria) is reported in bold. The $F_{3, \infty}$ column reports $p$-values under the null hypothesis of a uniform average performance across the four estimation criteria.

\begin{tabular}{|cc|ccccc|}
\hline \hline Model & & LS & SDLS & LNLS & QML & $F_{3, \infty}$ \\
\hline \hline MVAR & $(1,1)$ & 0.3113 & 0.2031 & $\mathbf{0 . 1 8 8 3}$ & 0.2105 & $<0.0001$ \\
& $(2,1)$ & 0.2891 & 0.1948 & $\mathbf{0 . 1 8 2 7}$ & 0.2052 & $<0.0001$ \\
MEXP & $(1,1)$ & 0.3067 & 0.2079 & $\mathbf{0 . 1 9 7 6}$ & 0.2228 & $<0.0001$ \\
& $(2,1)$ & 0.3247 & 0.2240 & $\mathbf{0 . 2 1 4 0}$ & 0.6067 & 0.3283 \\
MVOL & $(1,1)$ & 0.2842 & 0.1905 & $\mathbf{0 . 1 8 2 7}$ & 0.2036 & $<0.0001$ \\
& $(2,1)$ & 0.2663 & 0.1850 & $\mathbf{0 . 1 7 7 6}$ & 0.1985 & $<0.0001$ \\
MLOG & $(1,1)$ & 0.2559 & 0.1850 & $\mathbf{0 . 1 7 9 8}$ & 0.1990 & $<0.0001$ \\
& $(2,1)$ & 0.2559 & 0.1812 & $\mathbf{0 . 1 7 5 6}$ & 0.1945 & $<0.0001$ \\
HAR & & 0.2914 & 0.1961 & $\mathbf{0 . 1 8 2 9}$ & 0.2048 & $<0.0001$ \\
LOG-HAR & 0.2889 & 0.1857 & $\mathbf{0 . 1 7 8 5}$ & 0.1997 & $<0.0001$ \\
\hline \hline
\end{tabular}

\section{Table 20:}

Tantamount to Table 8 but with no Winsorization of IS data. QML forecasting loss for out-of-sample evaluations: for each of the four estimation criteria (LS, SDLS, LNLS and QML), in each row we report the average loss (by row 28 tickers times 6 periods $=168$ possible instances). For every specification, the lowest loss (across estimation criteria) is reported in bold. The $F_{3, \infty}$ column reports $p$-values under the null hypothesis of a uniform average performance across the four estimation criteria.

\begin{tabular}{|cc|ccccc|}
\hline \hline Model & & LS & SDLS & LNLS & QML & $F_{3, \infty}$ \\
\hline \hline \multirow{2}{*}{ MVAR } & $(1,1)$ & 1.1520 & 1.1226 & 1.1206 & $\mathbf{1 . 1 1 8 7}$ & $<0.0001$ \\
& $(2,1)$ & 1.1461 & 1.1211 & 1.1199 & $\mathbf{1 . 1 1 7 5}$ & $<0.0001$ \\
MEXP & $(1,1)$ & 1.1502 & 1.1270 & 1.1275 & $\mathbf{1 . 1 2 3 6}$ & $<0.0001$ \\
& $(2,1)$ & 1.1565 & 1.1321 & $\mathbf{1 . 1 3 1 9}$ & 1.1659 & 0.3698 \\
MVOL & $(1,1)$ & 1.1408 & 1.1181 & 1.1199 & $\mathbf{1 . 1 1 7 2}$ & $<0.0001$ \\
& $(2,1)$ & 1.1363 & 1.1176 & 1.1193 & $\mathbf{1 . 1 1 6 1}$ & $<0.0001$ \\
MLOG & $(1,1)$ & 1.1310 & $\mathbf{1 . 1 1 6 4}$ & 1.1201 & 1.1165 & $<0.0001$ \\
& $(2,1)$ & 1.1319 & 1.1162 & 1.1197 & $\mathbf{1 . 1 1 5 6}$ & $<0.0001$ \\
\multirow{2}{*}{ HAR } & & 1.1468 & 1.1213 & 1.1200 & $\mathbf{1 . 1 1 7 3}$ & $<0.0001$ \\
\multicolumn{2}{|c|}{ LOG-HAR } & 1.1452 & 1.1174 & 1.1205 & $\mathbf{1 . 1 1 7 2}$ & $<0.0001$ \\
\hline \hline
\end{tabular}




\section{Table 21:}

Tantamount to Table 9 but with no Winsorization of IS data. LS forecasting loss and estimation criterion. The first three columns report the percentage a specification is best: IS, in-sample (based on BIC); OOS, out-of-sample (LS loss); OOS|IS, best out-of-sample, limiting to the best in-sample. A-Loss and M-Loss are the average and median losses. The BIC row contains out-of-sample results for the best specifications selected in-sample by the information criterion. Individual A-Losses are tested against the corresponding value on the bottom row (a *, * $* * *$ signifies a better performance $-10 \%, 5 \%, 1 \%$ significance - the reverse for the corresponding $*, * *, * * *)$.

\begin{tabular}{|cc|ccccc|}
\hline \hline Model & & IS & OOS & OOS|IS & A-Loss & M-Loss \\
\hline \hline MVAR & $(1,1)$ & $0.00 \%$ & $1.79 \%$ & $0.00 \%$ & 3.2963 & 1.2859 \\
& $(2,1)$ & $0.00 \%$ & $7.74 \%$ & $0.00 \%$ & 3.2135 & 1.1525 \\
MEXP & $(1,1)$ & $0.00 \%$ & $0.60 \%$ & $0.00 \%$ & 7.2577 & 1.2632 \\
& $(2,1)$ & $0.60 \%$ & $0.00 \%$ & $0.00 \%$ & 9.1616 & 1.3764 \\
MVOL & $(1,1)$ & $0.00 \%$ & $1.19 \%$ & $0.00 \%$ & 3.2137 & 1.1717 \\
& $(2,1)$ & $0.00 \%$ & $9.52 \%$ & $0.00 \%$ & $3.1330^{*}$ & 1.1243 \\
MLOG & $(1,1)$ & $44.05 \%$ & $32.74 \%$ & $31.08 \%$ & $3.1247^{*}$ & 1.1647 \\
& $(2,1)$ & $39.29 \%$ & $27.38 \%$ & $16.67 \%$ & 3.1610 & 1.1457 \\
HAR & & $4.76 \%$ & $7.74 \%$ & $0.00 \%$ & 3.2463 & 1.1433 \\
LOG-HAR & $11.31 \%$ & $11.31 \%$ & $42.11 \%$ & 3.4370 & 1.1624 \\
\hline BIC & & & $25.00 \%$ & & 3.4519 & 1.1554 \\
\hline \hline
\end{tabular}

\section{Table 22:}

Tantamount to Table 10 but with no Winsorization of IS data. SDLS forecasting loss and estimation criterion. The first three columns report the percentage a specification is best: IS, in-sample (based on $B I C$ ); OOS, out-of-sample (SDLS loss); OOS|IS, best out-of-sample, limiting to the best in-sample. A-Loss and M-Loss are the average and median losses. The BIC row contains out-of-sample results for the best specifications selected in-sample by the information criterion. Individual A-Losses are tested against the corresponding value on the bottom row (a $*$, * $* * *$ signifies a better performance $-10 \%, 5 \%, 1 \%$ significance - the reverse for the corresponding $*, * *, * * *)$.

\begin{tabular}{|cc|ccccc|}
\hline \hline Model & & IS & OOS & OOS|IS & A-Loss & M-Loss \\
\hline \hline MVAR & $(1,1)$ & $0.00 \%$ & $0.00 \%$ & $0.00 \%$ & $0.1056_{* * *}$ & 0.0817 \\
& $(2,1)$ & $0.00 \%$ & $5.36 \%$ & $0.00 \%$ & $0.1016_{* * *}$ & 0.0776 \\
MEXP & $(1,1)$ & $0.00 \%$ & $1.19 \%$ & $0.00 \%$ & $0.1316_{* * *}$ & 0.0878 \\
& $(2,1)$ & $0.00 \%$ & $0.00 \%$ & $0.00 \%$ & $0.1408_{* * *}$ & 0.0953 \\
MVOL & $(1,1)$ & $0.00 \%$ & $0.00 \%$ & $0.00 \%$ & $0.0996_{* * *}$ & 0.0775 \\
& $(2,1)$ & $5.95 \%$ & $5.95 \%$ & $0.00 \%$ & 0.0972 & 0.0759 \\
MLOG & $(1,1)$ & $42.26 \%$ & $11.90 \%$ & $9.86 \%$ & 0.0965 & 0.0757 \\
& $(2,1)$ & $30.95 \%$ & $50.60 \%$ & $55.77 \%$ & $0.0949^{* * *}$ & 0.0752 \\
HAR & & $4.17 \%$ & $10.71 \%$ & $0.00 \%$ & $0.1022_{* * *}$ & 0.0775 \\
LOG-HAR & $16.67 \%$ & $14.29 \%$ & $7.14 \%$ & 0.0974 & 0.0752 \\
\hline \multicolumn{2}{|c|}{ BIC } & & $22.00 \%$ & & 0.0966 & 0.0757 \\
\hline \hline
\end{tabular}




\section{Table 23:}

Tantamount to Table 11 but with no Winsorization of IS data. LNLS forecasting loss and estimation criterion. The first three columns report the percentage a specification is best: IS, in-sample (based on BIC); OOS, out-of-sample (LNLS loss); OOS|IS, best out-of-sample, limiting to the best in-sample. A-Loss and M-Loss are the average and median losses. The BIC row contains out-of-sample results for the best specifications selected in-sample by the information criterion. Individual A-Losses are tested against the corresponding value on the bottom row (a *, * , $* * *$ signifies a better performance $-10 \%, 5 \%, 1 \%$ significance - the reverse for the corresponding $*, * *, * * *)$.

\begin{tabular}{|cc|ccccc|}
\hline \hline Model & & IS & OOS & OOS|IS & A-Loss & M-Loss \\
\hline \hline MVAR & $(1,1)$ & $0.00 \%$ & $0.00 \%$ & $0.00 \%$ & $0.1883_{* * *}$ & 0.1815 \\
& $(2,1)$ & $0.00 \%$ & $6.55 \%$ & $0.00 \%$ & $0.1827_{* * *}$ & 0.1761 \\
MEXP & $(1,1)$ & $0.00 \%$ & $0.00 \%$ & $0.00 \%$ & $0.1976_{* * *}$ & 0.1845 \\
& $(2,1)$ & $0.00 \%$ & $1.79 \%$ & $0.00 \%$ & $0.2140_{* * *}$ & 0.1974 \\
MVOL & $(1,1)$ & $0.00 \%$ & $0.60 \%$ & $0.00 \%$ & $0.1827_{* * *}$ & 0.1770 \\
& $(2,1)$ & $7.74 \%$ & $5.36 \%$ & $0.00 \%$ & $0.1776_{* * *}$ & 0.1724 \\
MLOG & $(1,1)$ & $1.79 \%$ & $10.12 \%$ & $0.00 \%$ & $0.1798_{* * *}$ & 0.1742 \\
& $(2,1)$ & $75.00 \%$ & $54.76 \%$ & $51.59 \%$ & $0.1756^{* * *}$ & 0.1699 \\
HAR & & $1.19 \%$ & $8.33 \%$ & $0.00 \%$ & $0.1829_{* * *}$ & 0.1752 \\
LOG-HAR & $14.29 \%$ & $12.50 \%$ & $8.33 \%$ & $0.1785_{* * *}$ & 0.1739 \\
\hline BIC & & & $39.00 \%$ & & 0.1763 & 0.1709 \\
\hline \hline
\end{tabular}

\section{Table 24:}

Tantamount to Table 12 but with no Winsorization of IS data. QML forecasting loss and estimation criterion. The first three columns report the percentage a specification is best: IS, in-sample (based on BIC); OOS, out-of-sample (QML loss); OOS|IS, best out-of-sample, limiting to the best in-sample. A-Loss and M-Loss are the average and median losses. The BIC row contains out-of-sample results for the best specifications selected in-sample by the information criterion. Individual A-Losses are tested against the corresponding value on the bottom row (a $*$, * $* * *$ signifies a better performance $-10 \%, 5 \%, 1 \%$ significance - the reverse for the corresponding $*, * *, * * *)$.

\begin{tabular}{|cc|ccccc|}
\hline \hline Model & & IS & OOS & OOS|IS & A-Loss & M-Loss \\
\hline \hline MVAR & $(1,1)$ & $6.55 \%$ & $2.38 \%$ & $0.00 \%$ & $1.1187_{* * *}$ & 1.0961 \\
& $(2,1)$ & $0.00 \%$ & $6.55 \%$ & $0.00 \%$ & 1.1175 & 1.0942 \\
MEXP & $(1,1)$ & $0.00 \%$ & $1.19 \%$ & $0.00 \%$ & $1.1236_{* * *}$ & 1.0993 \\
& $(2,1)$ & $0.00 \%$ & $1.19 \%$ & $0.00 \%$ & 1.1659 & 1.1048 \\
MVOL & $(1,1)$ & $16.07 \%$ & $1.79 \%$ & $0.00 \%$ & 1.1172 & 1.0939 \\
& $(2,1)$ & $0.00 \%$ & $4.76 \%$ & $0.00 \%$ & $1.1161^{* * *}$ & 1.0915 \\
MLOG & $(1,1)$ & $32.74 \%$ & $17.86 \%$ & $7.27 \%$ & $1.1165^{* * *}$ & 1.0933 \\
& $(2,1)$ & $2.38 \%$ & $34.52 \%$ & $0.00 \%$ & $1.1156^{* * *}$ & 1.0911 \\
HAR & & $13.10 \%$ & $22.62 \%$ & $9.09 \%$ & 1.1173 & 1.0942 \\
LOG-HAR & $29.17 \%$ & $7.14 \%$ & $4.08 \%$ & 1.1172 & 1.0916 \\
\hline BIC & & $4.00 \%$ & & 1.1176 & 1.0933 \\
\hline \hline
\end{tabular}


